\title{
Consensus Reaching with Time Constraints and Minimum Adjustments in Group with Bounded Confidence Effects
}

\author{
Haiming Liang, Yucheng Dong, Zhaogang Ding, Raquel Ureña, \\ Francisco Chiclana, Enrique Herrera-Viedma
}

\begin{abstract}
In the bounded confidence model it is widely known that individuals rely on the opinions of their close friends or people with similar interests. Meanwhile, the decision maker always hopes that the opinions of individuals can reach a consensus in a required time. Therefore, with this idea in mind, this paper develops a consensus reaching model with time constraints and minimum adjustments in a group with bounded confidence effects. In the proposed consensus approach, the minimum adjustments rule is used to modify the initial opinions of individuals with bounded confidence, which can further influence the opinion evolutions of individuals to reach a consensus in a required time. The properties of the model are studied, and detailed numerical examples and comparative simulation analysis are provided to justify its feasibility.
\end{abstract}

Index Terms-Consensus, time constraints, bounded confidence, minimum adjustments, opinion dynamics, group decision making

\section{INTRODUCTION}

Cons onsensus reaching is an important research problem that involves in the preference evolution and aggregation of a group of individuals [1-3], and it is present in a wide range of application areas [4-6], such as military [7], management [8], and so on. In spite of the fact consensus reaching does not necessarily imply that the

This work was supported by the grants (Nos. 71871149, 71571124, 71971149 and 71601133) from NSF of China, the grants (Nos. sksyl201705, 2018hhs-58 and YJ201906) from Sichuan University, and FEDER funds provided in the National Spanish project TIN2016-75850-R and EU project H2020-MSCA-IF-2016- DeciTrustNET-746398. (Corresponding author: Yucheng Dong.)

Haiming Liang and Yucheng Dong are with Business School, Sichuan University, Chengdu, China (e-mails: hmliang@scu.edu.cn (H. Liang), ycdong@scu.edu.cn (Y. Dong)).

Zhaogang Ding is with School of Public Management, Northwest University, Xi’an, China (e-mail: zgding@nwu.edu.cn (Z. Ding)).

Raquel Ureña is with Institute of Artificial Intelligence (IAI), School of Computer Science and Informatics, De Montfort University, Leicester, UK (e-mail: raquel.urena@dmu.ac.uk (R. Ureña)).

Francisco Chiclana is with Institute of Artificial Intelligence (IAI), School of Computer Science and Informatics, De Montfort University, Leicester, UK, and Department of Computer Science and Artificial Intelligence, University of Granada, Granada, Spain (e-mail: chiclana@ dmu.ac.uk, inv.chiclana@ugr.es (F. Chiclana)).

Enrique Herrera-Viedma is with Department of Computer Science and Artificial Intelligence, University of Granada, Granada, Spain, and Department of Electrical and Computer Engineering, Faculty of Engineering, King Abdulaziz University, Jeddah, Saudi Arabia (e-mail: viedma@decsai.ugr.es (E. Herrera-Viedma)). best solution will be obtained, the consensus reaching process has the following key advantages [9]: (i) Building connections among the decision makers. Using the consensus reaching process as a decision tool means taking the time to find unity on how to proceed before moving forward. It's a synthesizing process that promotes the communication among decision makers. (ii) More effective implementation. When decision makers' preferences and concerns are considered, they are much more likely to actively participate in the implementation of the obtained solution. More concretely, the conensus issues have been widely investigated in opinion dynamics (OD) and group decision making (GDM).

In the OD context the individuals express, interact and update their opinions until reaching an agreement. In the literature, a wide variety of opinion dynamics approaches have been proposed, among which, we can highlight the following: voter model [10, 11], Sznajd model [12] majority-rule model [13, 14], DeGroot model [15], Deffuant model [16], Hegselmann-Krause (HK) model [17], OCR model [18], continuous opinions and discrete action (CODA) models [19]. In addition to the above general opinion dynamics models, some scholars have attempted to extend the HK model [20, 21], discuss the strategy to support the consensus reaching [22] and the opinion control [23].

In the case of consensus applied to group decision making (GDM) contexts, individuals discuss and modify their preferences in order to reach a collective agreement before making decisions [24-27]. In this case, one of the main challenges, consists in designing an effective feedback mechanism to guide the individuals to reach consensus with minimum adjustments or costs [28-31]. In general, three kinds of rules have been proposed to measure costs in consensus reaching [32]: (1) the minimum distance rules, which minimize the distance between the original opinions and the adjusted opinions [33], (2) the maximum expert rules, which maximize the number of experts at an acceptable consensus level/degree with a limited budget [34, 35], and (3) the minimum account rules, which minimize the number of adjusted opinions when reaching consensus [36, 37].

Even though the existing approaches have made significant contributions on consensus reaching, there are still some challenges that require research attention:

(i) Opinion dynamics mainly focused on studying the evolution and diffusion among individuals. However, in the many occasions, consensus among individuals in the OD context may not be achieved resulting in a sort of opinion polarization or fragmentation. Moreover, time constraints 
become one of the main challenges in consensus reaching in practice. For example, in practical consensus reaching problem, such as, the salary assignment plan, introducing the new project, the individual income tax, the decision maker in the government or enterprise always hopes that the collected opinions of individuals can reach a consensus in a required time. Thus, it is necessary to design a mechanism to assist the individuals to reach a consensus in a determined time.

(ii) Consensus has been widely investigated in the GDM context. In the existing consensus studies in the GDM context, minimum adjustments or costs have become one of the key issues in designing the feedback mechanisms. However, the real evolution of the individuals' opinions are often neglected in the existing feedback mechanisms with minimum adjustments, while in practice individuals often share and evolve their opinions and preferences through the interactions. Thus, it is necessary to propose consensus reaching mechanisms that take into consideration these evolutions of opinions.

In the light of the shortcomings and challenges mentioned above, this contribution proposes a new consensus reaching approach that considers time constraints and minimum adjustments in a group with bounded confidence effects. In essence, the proposed model with minimum adjustments and time constraints (i.e., MAT model) modifies the initial opinions of the individuals that iteratively may influence the opinion evolutions of other individuals to reach a consensus in a determined time. The desirable properties of the MAT model are discussed. Finally, in order to test and validate the proposed model a numerical example and a comparison with another consensus model (i.e., GMAT model) have been carried out.

The rest of the paper is organized as follows: Section II introduces the basic knowledge regarding the HK model and the proposed problem. Then, Section III presents the MAT consensus model with bounded confidence effects. In Section IV, some desirable properties of the proposed MAT consensus model are discussed. Following this, Section V reports on a numerical example and a simulation analysis to illustrate the feasibility of the proposed approach. Finally, the conclusions are summarized in Section VI.

\section{PRELIMINARIES}

In order to facilitate the understanding and the use of the bounded confidence models, these models are briefly reviewed.

\section{A. The bounded confidence model}

The bounded confidence models assume that individuals only interact with each other when their opinions are similar, i.e. close. There exist two classical bounded confidence models: the DW model and the HK model [17]. In this paper, we adopt the HK model as the basic model.

Before introducing the $\mathrm{HK}$ model, we define some related notations. Let $A=\left\{A_{1}, A_{2}, \ldots, A_{N}\right\}$ be the set of individuals, where $A_{i}$ denotes the $i$ th individual, for $i=1,2, \ldots, N$. Let $x_{i}(t) \in[0,1]$ denote the opinion of individual $A_{i}$ at time $t$. Let $\varepsilon$ be the homogeneous of bounded confidence of the individuals.

The HK model is composed of the following steps:

Step 1 Determination of the confidence sets.

Let $I\left(A_{i}, X(t)\right)$ be the confidence set of individual $A_{i}$ at time $t$, where

$$
\begin{gathered}
I\left(A_{i}, X(t)\right)=\left\{A_{j}|| x_{i}(t)-x_{j}(t) \mid \leq \varepsilon\right\}, i=1,2, \ldots, N, \\
t=0,1,2, \ldots
\end{gathered}
$$

\section{Step 2 Calculation of the weight}

Let $\# I\left(A_{i}, X(t)\right)$ denote the cardinality of the set $I\left(A_{i}, X(t)\right)$. Let $w_{i j}(t)$ be the weight that individual $A_{i}$ assigns to individual $A_{j}$ at time $t$, where

$$
\begin{aligned}
w_{i j}(t) & =\left\{\begin{array}{cc}
\frac{1}{\# I\left(A_{i}, X(t)\right)}, & A_{j} \in I\left(A_{i}, X(t)\right) \\
0, & A_{j} \notin I\left(A_{i}, X(t)\right)
\end{array},\right. \\
i & =1,2, \ldots, N, t=0,1,2, \ldots
\end{aligned}
$$

Clearly, $w_{i j}(t) \geq 0$ and $\sum_{j=1}^{N} w_{i j}(t)=1$.

\section{Step 3 Evolution of the opinions}

The evolutions of the opinions in the HK model are modelled as the weighted arithmetic means of opinions of individuals in the confidence sets, i.e.,

$$
\begin{gathered}
x_{i}(t+1)=\frac{\sum_{A_{j} \in I\left(A_{i}, X(t)\right)} x_{j}(t)}{\# I\left(A_{i}, X(t)\right)}=w_{i 1}(t) x_{1}(t)+ \\
w_{i 2}(t) x_{2}(t)+\cdots+w_{i N}(t) x_{N}(t), \\
i=1,2, \ldots, N .
\end{gathered}
$$

B. The proposed problem: Consensus reaching with time constraints and minimum adjustments in a group

In this contribution consensus is referred to the state in which all the individuals reach a full and unanimous agreement. Specifically, let $A_{i}, A_{j} \in A$ be any two individuals. The consensus among the individuals will be reached at time $t$ if $x_{i}(t)=x_{j}(t)$, for all $i, j=1,2, \ldots, N$.

In opinion dynamics context, the individuals continuously update their opinions before reaching the stable state. However, in the majority of the occasions the consensus among individuals is not reachable resulting in fragmentation or polarization. To demonstrate these different formats of opinions in the stable state, 3 sets of individuals' initial opinions are generated, and their opinion evolutions based on the HK model are presented in Fig. 1.

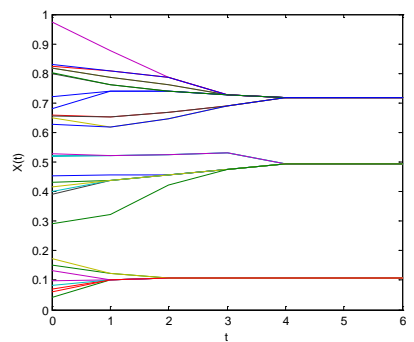

(a) Fragmentation

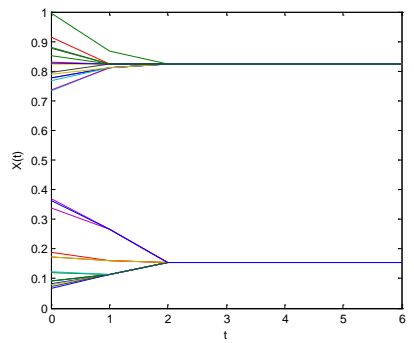

(b) Polarization

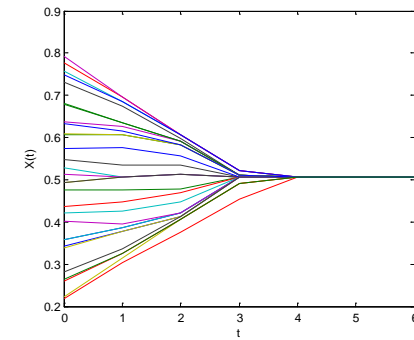

(c) Consensus

Fig. 1. Three stable states in opinion dynamics 
In this contribution we propose a consensus model with time constraints and minimum adjustments that modify the initial opinions of individuals to further influence the opinion evolutions of the others individuals that aim to reach a consensus in a required time.

Additional main notations used in this paper are listed as follows:

$t$ : the discrete time, $t=0,1,2, \ldots$;

$t^{*}$ : the determined time when all the individuals should reach a consensus;

$x_{i}(0)$ : the original opinion of individual $A_{i}$ in the initial time, where $x_{i}(0) \in[0,1]$;

$\bar{x}_{i}(0)$ : the adjusted opinion of individual $A_{i}$ in the initial time, where $\bar{x}_{i}(0) \in[0,1]$;

$\bar{x}_{i}(t)$ : the opinion that $\bar{x}_{i}(0)$ is evolved to time $t$, where $\bar{x}_{i}(t) \in[0,1]$, for $t=1,2, \ldots, t^{*}$;

\section{THE CONSENSUS MODEL WITH BOUNDED CONFIDENCE EFFECTS}

In this section the new consensus model with bounded confidence effects, referred to as the MAT model, is introduced. The equivalent forms of the MAT model at different time constraints are presented as well. Furthermore with the objective of solving the proposed MAT model a particle swarm optimization (PSO) algorithm is designed.

\section{A. The formulation of the consensus model}

The aim is to reach a consensus in a group with bounded confidence effects by minimizing the adjustments of the initial opinions of individuals, i.e.,

$$
\operatorname{Min} \sum_{i=1}^{N}\left|\bar{x}_{i}(0)-x_{i}(0)\right|
$$

The adjusted initial opinions of individuals evolve following the HK model. Thus, let $\bar{x}_{i}(t)$ be as defined before, then

$$
\begin{aligned}
& \bar{x}_{i}(t+1)=\frac{\sum_{A_{j} \in I\left(A_{i}, \bar{X}(t)\right)} \bar{x}_{j}(t)}{\# I\left(A_{i}, \bar{X}(t)\right)}, \\
& i=1,2, \ldots, N, t=0,1,2, \ldots, t^{*}-1
\end{aligned}
$$

where $\quad I\left(A_{i}, \bar{X}(t)\right)=\left\{A_{j}|| \bar{x}_{i}(t)-\bar{x}_{j}(t) \mid \leq \varepsilon\right\} \quad, \quad$ and $\# I\left(A_{i}, \bar{X}(t)\right)$ denotes its cardinality.

Because the opinion of all the individuals must reach a consensus in a required time $t^{*}$, we have:

$$
\bar{x}_{i}\left(t^{*}\right)=\bar{x}_{j}\left(t^{*}\right), \quad i, j=1,2, \ldots, N
$$

As a result, based on Eqs. (4)-(6), the consensus model is constructed as follows:

$$
\left\{\begin{array}{l}
\operatorname{Min} \sum_{i=1}^{N}\left|\bar{x}_{i}(0)-x_{i}(0)\right| \\
\bar{x}_{i}(t+1)=\frac{\sum_{A_{j} \in I\left(A_{i}, \bar{X}(t)\right)} \bar{x}_{j}(t)}{\# I\left(A_{i}, \bar{X}(t)\right)} \\
I\left(A_{i}, \bar{X}(t)\right)=\left\{\left|\bar{x}_{i}(t)-\bar{x}_{j}(t)\right| \leq \varepsilon\right\} \\
\bar{x}_{i}\left(t^{*}\right)=\bar{x}_{j}\left(t^{*}\right) \\
\bar{x}_{i}(0) \in[0,1]
\end{array}\right.
$$

where $\bar{x}_{i}(0)(i=1,2, \ldots, N)$ are decision variables in model (7).

Let $r_{i j}^{(t)}$ be the following $0-1$ variable indicating whether individual $A_{j}$ belongs to the set $I\left(A_{i}, \bar{X}(t)\right)$, i.e.,

$$
r_{i j}^{(t)}=\left\{\begin{array}{l}
1,\left|\bar{x}_{i}(t)-\bar{x}_{j}(t)\right| \leq \varepsilon, \\
0, \text { otherwise } \\
\quad i, j=1,2, \ldots, N, i \neq j
\end{array}\right.
$$

Clearly, $\quad \sum_{j=1}^{N} r_{i j}^{(t)}$ denotes the cardinality of
$I\left(A_{i}, \bar{X}(t)\right.$. ), i.e., $\sum_{j=1}^{N} r_{i j}^{(t)}=\# I\left(A_{i}, \bar{X}(t)\right)$. Then, Eq. (5) is rewritten as:

$$
\begin{gathered}
\bar{x}_{i}(t+1)=\frac{\sum_{j=1}^{N} r_{i j}^{(t)} \bar{x}_{j}(t)}{\sum_{j=1}^{N} r_{i j}^{(t)}}, \quad i=1,2, \ldots, N, t= \\
0,1,2, \ldots, t^{*}-1
\end{gathered}
$$

Furthermore, model (7) can be transformed as follows:

$$
\left\{\begin{array}{l}
\operatorname{Min} \sum_{i=1}^{N}\left|\bar{x}_{i}(0)-x_{i}(0)\right| \\
\bar{x}_{i}(t+1)=\frac{\sum_{j=1}^{N} r_{i j}^{(t)} \bar{j}_{j}(t)}{\sum_{j=1}^{N} r_{i j}^{(t)}} \\
\bar{x}_{i}\left(t^{*}\right)=\bar{x}_{j}\left(t^{*}\right) \\
r_{i j}^{(t)}=\left\{\begin{array}{l}
1,\left|\bar{x}_{i}(t)-\bar{x}_{j}(t)\right| \leq \varepsilon \\
0, \text { otherwise }
\end{array}\right. \\
\bar{x}_{i}(0) \in[0,1]
\end{array}\right.
$$

Thus, we can solve model (10) to obtain the solutions of model (7). In this study, to facilitate notations, we call model (10) the MAT model.

\section{B. Solving MAT model at different time constraints}

To obtain the optimal solutions of MAT model, we present its equivalent forms at different time constraints below:

Theorem 1. When $t^{*}=0$, the MAT model can be equivalently transformed as follows:

$$
\left\{\begin{array}{l}
\operatorname{Min} \sum_{i=1}^{N}\left|\bar{x}_{i}(0)-x_{i}(0)\right| \\
\bar{x}_{i}(0)=\bar{x}_{j}(0), i, j=1,2, \ldots, N, i \neq j \\
\bar{x}_{i}(0), \bar{x}_{j}(0) \in[0,1]
\end{array}\right.
$$

Theorem 2. When $t^{*}=1$, the MAT model can be equivalently transformed as follows:

$$
\left\{\begin{array}{l}
\operatorname{Min} \sum_{i=1}^{N}\left|\bar{x}_{i}(0)-x_{i}(0)\right| \\
\left|\bar{x}_{i}(0)-\bar{x}_{j}(0)\right| \leq \varepsilon, i, j=1,2, \ldots, N, i \neq j \\
\bar{x}_{i}(0), \bar{x}_{j}(0) \in[0,1]
\end{array}\right.
$$

Since Theorems 1 and 2 can be easily proved, their proofs of Theorems 1 and 2 are omitted in this paper.

Let $y_{i j}^{(t)}=\left\{\begin{array}{l}1, \bar{x}_{j}(t)-\bar{x}_{i}(t)>\varepsilon \\ 0, \bar{x}_{j}(t)-\bar{x}_{i}(t)<-\varepsilon\end{array}\right.$, then we have:

Theorem 3. When $t^{*}=2$, the MAT model can be equivalently transformed as follows:

$$
\left\{\begin{array}{l}
\operatorname{Min} \sum_{i=1}^{N}\left|\bar{x}_{i}(0)-x_{i}(0)\right| \\
\bar{x}_{i}(1) \sum_{j=1}^{N} r_{i j}^{(0)}=\sum_{j=1}^{N} r_{i j}^{(0)} \bar{x}_{j}(0) \\
\left|\bar{x}_{i}(1)-\bar{x}_{j}(1)\right| \leq \varepsilon, \\
\bar{x}_{j}(0)-\bar{x}_{i}(0) \geq-\varepsilon-\left(1-r_{i j}^{(0)}\right) M, \\
\bar{x}_{j}(0)-\bar{x}_{i}(0) \leq \varepsilon+\left(1-r_{i j}^{(0)}\right) M, \\
\bar{x}_{j}(0)-\bar{x}_{i}(0)<-\varepsilon+\left(y_{i j}^{(0)}+r_{i j}^{(0)}\right) M, \\
\bar{x}_{j}(0)-\bar{x}_{i}(0)>\varepsilon-\left(1-y_{i j}^{(0)}+r_{i j}^{(0)}\right) M, \\
y_{i j}^{(0)}+r_{i j}^{(0)}<2 \\
y_{i j}^{(0)}-r_{i j}^{(0)} \geq 0 \\
\bar{x}_{i}(0), \bar{x}_{j}(0) \in[0,1], y_{i j}^{(0)}, r_{i j}^{(0)} \in\{0,1\}
\end{array}\right.
$$

The proof of Theorem 3 is provided in Appendix A.

Models (11)-(13) are nonlinear programming models in which the constraints for dynamics evolution of opinions are not included. It is clear that models (11)-(13) can be easily solved using the lingo software package 11.0.

Theorem 4. Let $\bar{x}_{\sigma(i)}(0)$ be the $i$ th smallest value in the set $\left\{\bar{x}_{1}(0), \bar{x}_{2}(0), \ldots, \bar{x}_{N}(0)\right\}$. When $t^{*} \geq 3$, MAT 
model can be equivalently transformed as follows:

$$
\left\{\begin{array}{l}
\operatorname{Min} \sum_{i=1}^{N}\left|\bar{x}_{i}(0)-x_{i}(0)\right| \\
\bar{x}_{\sigma(i+1)}(0)-\bar{x}_{\sigma(i)}(0) \leq \varepsilon, \\
\bar{x}_{\sigma(i+1)}(0)-\bar{x}_{\sigma(i)}(0) \geq 0, \\
\bar{x}_{i}(t+1)=\frac{\sum_{j=1}^{N} r_{i j}^{(t)} \bar{x}_{j}(t)}{\sum_{j=1}^{N} r_{i j}^{(t)}} \\
\bar{x}_{i}\left(t^{*}\right)=\bar{x}_{j}\left(t^{*}\right) \\
r_{i j}^{(t)}=\left\{\begin{array}{l}
1,\left|\bar{x}_{i}(t)-\bar{x}_{j}(t)\right| \leq \varepsilon \\
0, \text { otherwise }
\end{array}\right.
\end{array}\right.
$$

The proof of Theorem 4 is provided in Appendix B.

In model (14), $\bar{x}_{\sigma(i+1)}(0)-\bar{x}_{\sigma(i)}(0) \leq \varepsilon$ and $\bar{x}_{\sigma(i+1)}(0)-\bar{x}_{\sigma(i)}(0) \geq 0$, for $i=1,2, \ldots, N-1$, are the necessary conditions for reaching a consensus [20], which can restrict the feasible region of solutions. Thus, we transform the MAT model into model (14).

In this paper, model (14) is a nonlinear programming model in which the constraints for dynamics evolution of opinions (third constraint in model (14)) are included. To solve model (14), we design an algorithm based on the classical PSO (Particle Swarm Optimization) algorithm below.

\section{The PSO algorithm I}

The PSO algorithm is a population based stochastic optimization technique inspired by bird blocking and fish schooling, proposed by Kennedy and Eberhart in [38]. In the PSO algorithm, each member, named particle, determines its position by combining the history of its own best location with those of others members of the swarm [38]. Based on this idea, in this contribution we design a new PSO algorithm I to solve model (14).

Following the general framework of the classical PSO algorithm, the key steps in the proposed PSO algorithm I can be described as follows:

\section{(1) Initiation}

Since $\bar{X}=\left(\bar{x}_{1}(0), \bar{x}_{2}(0), \ldots, \bar{x}_{N}(0)\right)$ is the solution vector in model (14), thus the position of each particle will have $N$ dimensions.

Let $P=\left\{P_{1}, P_{2}, \ldots, P_{N L}\right\}$ be the set of particles, where $P_{q}$ denotes the $q$ th particle, for $q=1,2, \ldots, N L$. Let $X_{q}^{l}(0)=\left(x_{q 1}^{l}(0), x_{q 2}^{l}(0), \ldots, x_{q N}^{l}(0)\right)$ be the position vector of $P_{q}$ at the initial time of the $l$ th iteration, where $x_{q h}^{l}(0)$ denotes the $h$ th dimension position of $P_{q}$ at the initial time of the $l$ th iteration.

Without loss of generality, suppose $x_{1}(0) \leq x_{2}(0) \leq$ $\cdots \leq x_{N}(0)$. Let $c l$ be the threshold value for clustering the opinions. Specifically, for two opinions $x_{i}(0)$ and $x_{i+1}(0)$, if $x_{i+1}(0)-x_{i}(0) \leq c l$, then we regard that individuals $A_{i}$ and $A_{i+1}$ belong to one opinion cluster. Then, the individuals will be divided into $e$ clusters $s_{1}, s_{2}, \ldots, s_{e}$. We use $\# s_{1}, \# s_{2}, \ldots, \# s_{e}$ to denote their cardinalities.

Assume $s_{y}=\left\{A_{y_{1}}, A_{y_{1}+1}, \ldots, A_{y_{1}+\# s_{y}-1}\right\}$, where $\# s_{y}=\max \left\{\# s_{1}, \# s_{2}, \ldots, \# s_{e}\right\}$ and $y \leq e$. Let $\operatorname{rand}(1)$ denote a random number in the interval $[0,1]$. Then, we have:

$$
\begin{gathered}
x_{q h}^{1}(0)=x_{h}(0), h=y_{1}, \ldots, y_{1}+\# s_{y}-1 \\
x_{q(h-1)}^{1}(0)=x_{q h}^{1}(0)-c l \times \operatorname{rand}(1), \\
h=2,3, \ldots, y_{1}
\end{gathered}
$$

$$
\begin{aligned}
& x_{q(h+1)}^{1}(0)=x_{q h}^{1}(0)+c l \times \operatorname{rand}(1), \\
& \quad h=y_{1}+\# s_{y}-1, \ldots, N-1
\end{aligned}
$$

Let $v_{q}^{l}=\left(v_{q 1}^{l}, v_{q 2}^{l}, \ldots, v_{q N}^{l}\right)$ be the velocity vector of particle $P_{q}$ in the $l$ th iteration, where $v_{q h}^{l}$ denotes the $h$ th dimension velocity of particle $P_{q}$, and $v_{q h}^{0}$ is a random number selected from $[0, \varepsilon]$.

\section{(2) Fitness function}

Let $x_{q h}^{l}(t+1)$ be the $h$ th dimension position of $P_{q}$ in time $t+1$ of $l$ th iteration, where

$$
x_{q h}^{l}(t+1)=\frac{\sum_{A_{j} \in I\left(A_{h}, X(t)\right)} x_{q j}^{l}(t)}{\# I\left(A_{h}, X(t)\right)}, q=1,2, \ldots, N
$$

where $I\left(A_{h}, X(t)\right)=\left\{A_{j}|| x_{q h}^{l}(t)-x_{q j}^{l}(t) \mid \leq \varepsilon\right\}$ and $\# I\left(A_{h}, X(t)\right)$ denotes its cardinality.

Let $f\left(X_{q}^{l}(0)\right)$ be the fitness function of particle $P_{q}$ in the $l$ th iteration, where

$$
\begin{gathered}
f\left(X_{q}^{l}(0)\right)= \\
\begin{cases}\sum_{i=1}^{N}\left|x_{q i}^{l}(0)-x_{i}(0)\right|, & x_{q 1}^{l}\left(t^{*}\right)=\cdots=x_{q N}^{l}\left(t^{*}\right) \\
M, & \text { otherwise }\end{cases}
\end{gathered}
$$

where $M$ denotes a large number.

\section{(3) Updating the velocity and position of particles}

Let $z_{p q}$ be the local best position of particle $P_{q}$, and let $z_{g}$ be the global best position. Then, $z_{p q}$ and $z_{g}$ can be determined as follows:

$$
\begin{aligned}
z_{p q} & =\arg \min _{x \in\left\{X_{q}^{1}(0), X_{q}^{2}(0), \ldots, X_{q}^{l}(0)\right\}} f(x) \\
z_{g} & =\arg \min _{x \in\left\{z_{p 1}, z_{p 2}, \ldots, z_{p N L}\right\}} f(x)
\end{aligned}
$$

Let $l_{\max }$ be the maximum iteration (number). When $l<l_{\text {max }}$, then the velocity and position of particles will be updated. Let $v_{q}^{l+1}=\left(v_{q 1}^{l+1}, v_{q 2}^{l+1}, \ldots, v_{q N}^{l+1}\right)$ be the velocity vector of particle $P_{q}$ in the $(l+1)$ th iteration, and let $X_{q}^{l+1}(0)=\left(x_{q 1}^{l+1}(0), x_{q 2}^{l+1}(0), \ldots, x_{q N}^{l+1}(0)\right)$ be the position vector of $P_{q}$ in the initial time of $(l+1)$ th iteration. Then, $v_{q}^{l+1}$ and $X_{q}^{l+1}(0)$ can be determined as follows:

$$
\begin{gathered}
v_{q}^{l+1}=w v_{q}^{l}+c_{1} r_{1}\left(z_{p q}-X_{q}^{l}(0)\right)+c_{2} r_{2}\left(z_{g}-X_{q}^{l}(0)\right) \\
X_{q}^{l+1}(0)=X_{q}^{l}(0)+v_{q}^{l+1}
\end{gathered}
$$

where $w$ denotes the inertia coefficient, $r_{1}$ and $r_{2}$ are random numbers drawn from the uniform distribution over the $[0,1]$ interval, $c_{1}$ and $c_{2}$ denotes the acceleration coefficient.

When $l=l_{\max }$, then we output the optimal solution $z_{g}$ and the optimal fitness degree $f\left(z_{g}\right)$.

Note 1. Compared with the classical PSO algorithm, the initial positions of particles in the proposed PSO algorithm I are generated by considering the necessary conditions of reaching a consensus (i.e., $\varepsilon$-profile) and the adjustments of opinions (i.e., the majority of individuals without adjustments). Obviously, the quality of the initial positions of particles in PSO algorithm I is higher than that in the classical PSO algorithm, which provides a basis for obtaining the optimal solutions.

\section{IV.DESIRABLE PROPERTIES OF THE MAT MODEL}

In this subsection, we introduce some desirable properties of MAT model. Some special cases, which the initial opinions of individuals will evolve to reach a consensus without modifying their initial opinions, are discussed.

Property 1. Let $t_{1}$ and $t_{2}$ be any two times, and let $A D\left(t_{1}\right)$ and $A D\left(t_{2}\right)$ be the adjustments of initial opinions 
in MAT model when $t^{*}=t_{1}$ and $t^{*}=t_{2}$. If $t_{1}<t_{2}$, then $A D\left(t_{1}\right)>A D\left(t_{2}\right)$.

The proof of Property 1 is provided in Appendix C.

Property 1 indicates that a shorter time for reaching consensus requires higher adjustments of the individuals' initial opinions.

Property 2. Let $x_{i}(0)$ and $x_{j}(0)$ be the original initial opinions of individuals $A_{i}$ and $A_{j}$. If $x_{i}(0) \leq$ $x_{j}(0)$, then it is $\bar{x}_{i}(0) \leq \bar{x}_{j}(0)$.

The proof of Property 2 can be seen in Appendix D.

Property 2 indicates that the initial opinions sequence consistency will still be maintained after modifying the initial opinions.

Property 3. Let $x_{\sigma(i)}(0)$ be as previously defined. When $N \leq 4$, if $0 \leq x_{\sigma(i+1)}(0)-x_{\sigma(i)}(0) \leq \varepsilon$, for $i=1,2, \ldots, N-1$, then the time required for reaching consensus will be smaller than 5 , i.e., $t^{*} \leq 5$.

The proof of Property 3 is provided in Appendix E.

Property 4. Let $x_{\sigma(i)}(0)$ be as previously defined. If $x_{\sigma(2)}(0)-x_{\sigma(1)}(0) \leq \varepsilon, x_{\sigma(N)}(0)-x_{\sigma(N-1)}(0) \leq \varepsilon$ and $\frac{x_{\sigma(N)}(0)+x_{\sigma(N-1)}(0)}{2}-\frac{x_{\sigma(2)}(0)+x_{\sigma(1)}(0)}{2} \leq \varepsilon$, then the consensus among the individuals will be reached at time $t^{*}=2$.

The proof of Property 4 is provided in Appendix F.

Property 5. Let $x_{\sigma(i)}(0)$ be as defined before. If there exists an individual $A_{k}$, such that:

(i) $\left|x_{\sigma(1)}(0)-x_{k}(0)\right| \leq \varepsilon$ and $\left|x_{\sigma(N)}(0)-x_{k}(0)\right| \leq \varepsilon$;

(ii) $\left|x_{\sigma(1)}(1)-x_{k}(1)\right| \leq\left|x_{\sigma(1)}(0)-x_{k}(0)\right|$, and
$\left|x_{\sigma(N)}(1)-x_{k}(1)\right| \leq\left|x_{\sigma(N)}(0)-x_{k}(0)\right|$.

Then, consensus among the individuals will be reached.

The proof of Property 5 is provided in Appendix G.

\section{NUMERICAL AND SIMULATION ANALYSIS}

This section presents a numerical example to show the usefulness of the MAT model. A comparison with another consensus model (GMAT model) is also provided, and finally an analysis to display the performance of the MAT model is presented.

\section{A. The numerical example}

BR Co. Ltd. is a company in the area of poultry-processing that wants to collect the opinions of workers over the necessity of regular production. A survey was carried out among employees where they were asked to provide their opinions expressed by a numerical value in the interval $[0,1]$, where ' 0 ' denotes the extremely unnecessary, and ' 1 ' denotes the extremely necessary.

The BR company hopes that the opinions of its worker can reach the consensus in a limited period of time. In this paper, we consider two cases of the initial opinions of 30 workers.

Case I: The fragmentation case

In this case, the required time $t^{*}$ is set as 4 , i.e., $t^{*}=4$, with the initial opinions of individuals are given in Table 1 .

Table 1. The initial opinions of 30 workers

\begin{tabular}{c|c|c|c|c|c|c|c|c|c}
\hline$x_{1}(0)$ & $x_{2}(0)$ & $x_{3}(0)$ & $x_{4}(0)$ & $x_{5}(0)$ & $x_{6}(0)$ & $x_{7}(0)$ & $x_{8}(0)$ & $x_{9}(0)$ & $x_{10}(0)$ \\
\hline 0.65 & 0.21 & 0.50 & 0.62 & 0.87 & 0.94 & 0.53 & 0.18 & 0.16 & 0.24 \\
\hline$x_{11}(0)$ & $x_{12}(0)$ & $x_{13}(0)$ & $x_{14}(0)$ & $x_{15}(0)$ & $x_{16}(0)$ & $x_{17}(0)$ & $x_{18}(0)$ & $x_{19}(0)$ & $x_{20}(0)$ \\
\hline 0.84 & 0.22 & 0.80 & 0.35 & 0.89 & 0.41 & 0.19 & 0.35 & 0.57 & 0.48 \\
\hline$x_{21}(0)$ & $x_{22}(0)$ & $x_{23}(0)$ & $x_{24}(0)$ & $x_{25}(0)$ & $x_{26}(0)$ & $x_{27}(0)$ & $x_{28}(0)$ & $x_{29}(0)$ & $x_{30}(0)$ \\
\hline 0.41 & 0.81 & 0.55 & 0.53 & 0.92 & 0.37 & 0.65 & 0.65 & 0.43 & 0.55 \\
\hline
\end{tabular}

Let $\varepsilon=0.2$. Based on the HK model, the evolutions of the opinions of these 30 workers are shown in Fig. 2

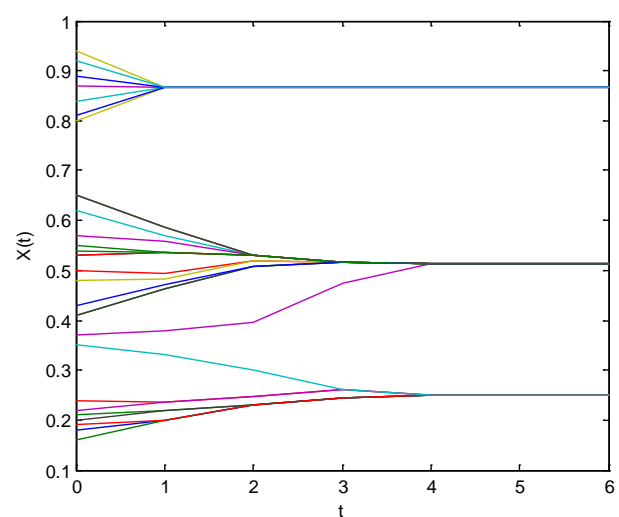

Fig. 2. The evolution of the initial opinions under Case I

From Fig. 2, the opinions of individuals in the stable state will form three clusters.

Thus, we need to use the proposed method to reach a consensus. Since $t^{*}=4$, the MAT model is constructed as follows:

$$
\left\{\begin{array}{l}
\operatorname{Min} \sum_{i=1}^{30}\left|\bar{x}_{i}(0)-x_{i}(0)\right| \\
\bar{x}_{\sigma(i+1)}(0)-\bar{x}_{\sigma(i)}(0) \leq 0.2 \\
\bar{x}_{\sigma(i+1)}(0)-\bar{x}_{\sigma(i)}(0) \geq 0 \\
\bar{x}_{i}(t+1)=\frac{\sum_{j=1}^{30} r_{i j}^{(t)} \bar{x}_{j}(t)}{\sum_{j=1}^{30} r_{i j}^{(t)}} \\
\bar{x}_{i}(4)=\bar{x}_{j}(4)
\end{array}\right.
$$

Let the maximum number of iterations $l_{\max }=500$, $c l=0.1, w=0.2$ and $c_{1}=c_{2}=2$. The size of the swarm consists of 100 particles. Then, we use the PSO algorithm I to solve the consensus model. The fitness degree in successive generations is depicted in Fig. 3. 


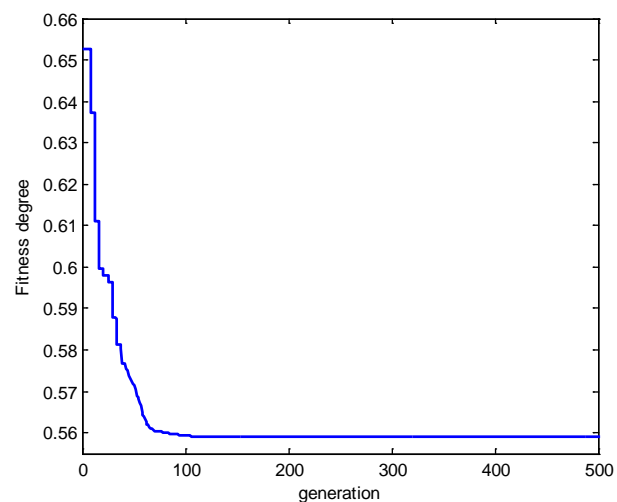

Fig. 3. The fitness degree in successive generations under Case I

The adjusted initial opinions are listed in Table 2 . Then, based on the HK model, the adjusted initial opinions of these 30 workers will evolve to reach a consensus at time $t^{*}=4$, that is in four iterations as depicted in Fig. 4 .

Table 2. The optimal opinions of 30 workers

\begin{tabular}{c|c|c|c|c|c|c|c|c|c}
\hline $\bar{x}_{1}(0)$ & $\bar{x}_{2}(0)$ & $\bar{x}_{3}(0)$ & $\bar{x}_{4}(0)$ & $\bar{x}_{5}(0)$ & $\bar{x}_{6}(0)$ & $\bar{x}_{7}(0)$ & $\bar{x}_{8}(0)$ & $\bar{x}_{9}(0)$ & $\bar{x}_{10}(0)$ \\
\hline 0.65 & 0.2443 & 0.50 & 0.62 & 0.8289 & 0.9067 & 0.53 & 0.178 & 0.16 & 0.3157 \\
\hline $\bar{x}_{11}(0)$ & $\bar{x}_{12}(0)$ & $\bar{x}_{13}(0)$ & $\bar{x}_{14}(0)$ & $\bar{x}_{15}(0)$ & $\bar{x}_{16}(0)$ & $\bar{x}_{17}(0)$ & $\bar{x}_{18}(0)$ & $\bar{x}_{19}(0)$ & $\bar{x}_{20}(0)$ \\
\hline 0.65 & 0.2741 & 0.7012 & 0.35 & 0.8498 & 0.41 & 0.208 & 0.35 & 0.57 & 0.48 \\
\hline $\bar{x}_{21}(0)$ & $\bar{x}_{22}(0)$ & $\bar{x}_{23}(0)$ & $\bar{x}_{24}(0)$ & $\bar{x}_{25}(0)$ & $\bar{x}_{26}(0)$ & $\bar{x}_{27}(0)$ & $\bar{x}_{28}(0)$ & $\bar{x}_{29}(0)$ & $\bar{x}_{30}(0)$ \\
\hline 0.65 & 0.7494 & 0.55 & 0.53 & 0.8757 & 0.37 & 0.65 & 0.65 & 0.43 & 0.55 \\
\hline
\end{tabular}

(ii) The polarization case

In this case, the required time $t^{*}$ is set as 5 , i.e.,

$t^{*}=5$, with the initial opinions of individuals are given in Table 3 .

Table 3. The initial opinions of 30 workers

\begin{tabular}{c|c|c|c|c|c|c|c|c|c}
\hline$x_{1}(0)$ & $x_{2}(0)$ & $x_{3}(0)$ & $x_{4}(0)$ & $x_{5}(0)$ & $x_{6}(0)$ & $x_{7}(0)$ & $x_{8}(0)$ & $x_{9}(0)$ & $x_{10}(0)$ \\
\hline 0.07 & 0.05 & 0.08 & 0.04 & 0.06 & 0.12 & 0.04 & 0.15 & 0.03 & 0.06 \\
\hline$x_{11}(0)$ & $x_{12}(0)$ & $x_{13}(0)$ & $x_{14}(0)$ & $x_{15}(0)$ & $x_{16}(0)$ & $x_{17}(0)$ & $x_{18}(0)$ & $x_{19}(0)$ & $x_{20}(0)$ \\
\hline 0.17 & 0.23 & 0.06 & 0.03 & 0.14 & 0.29 & 0.88 & 0.89 & 0.96 & 0.94 \\
\hline$x_{21}(0)$ & $x_{22}(0)$ & $x_{23}(0)$ & $x_{24}(0)$ & $x_{25}(0)$ & $x_{26}(0)$ & $x_{27}(0)$ & $x_{28}(0)$ & $x_{29}(0)$ & $x_{30}(0)$ \\
\hline 0.88 & 0.94 & 0.90 & 0.85 & 0.94 & 0.92 & 0.98 & 0.95 & 0.97 & 0.95 \\
\hline
\end{tabular}

Let $\varepsilon=0.2$. Based on the HK model, the evolutions of initial opinions of 30 workers are depicted in Fig. 5.

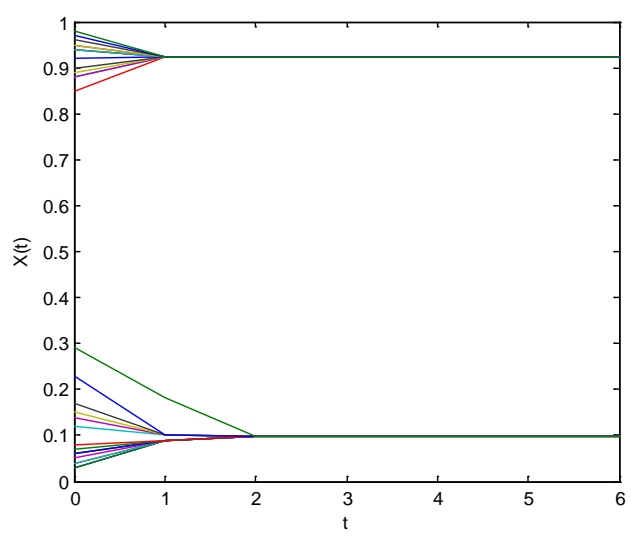

Fig. 5. The evolution of the initial opinions under Case II

As we can observe in Fig. 5, the opinions of individuals in the stable state will reach a polarization state. Thus, the proposed method to reach a consensus in required

Since $t^{*}=5$, then the MAT model can be constructed. Let the maximum number of iterations $l_{\max }=500$, $w=0.2, c_{1}=c_{2}=2$ and $c l=0.1$. The size of the swarm consists of 100 particles. Then, we use the PSO algorithm I to solve the consensus model. The fitness degree in successive generations is depicted in Fig. 6.

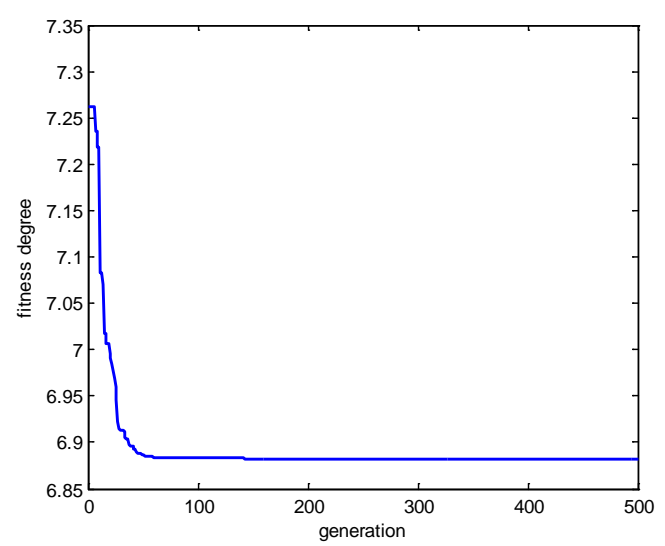

Fig. 6. The fitness degree in successive generations under Case II 
Table 4. The adjusted opinions of 30 workers

\begin{tabular}{c|c|c|c|c|c|c|c|c|c}
\hline $\bar{x}_{1}(0)$ & $\bar{x}_{2}(0)$ & $\bar{x}_{3}(0)$ & $\bar{x}_{4}(0)$ & $\bar{x}_{5}(0)$ & $\bar{x}_{6}(0)$ & $\bar{x}_{7}(0)$ & $\bar{x}_{8}(0)$ & $\bar{x}_{9}(0)$ & $\bar{x}_{10}(0)$ \\
\hline 0.1558 & 0.1177 & 0.1614 & 0.1123 & 0.1424 & 0.1625 & 0.0944 & 0.1976 & 0.03 & 0.1497 \\
\hline $\bar{x}_{11}(0)$ & $\bar{x}_{12}(0)$ & $\bar{x}_{13}(0)$ & $\bar{x}_{14}(0)$ & $\bar{x}_{15}(0)$ & $\bar{x}_{16}(0)$ & $\bar{x}_{17}(0)$ & $\bar{x}_{18}(0)$ & $\bar{x}_{19}(0)$ & $\bar{x}_{20}(0)$ \\
\hline 0.3050 & 0.3177 & 0.1558 & 0.0392 & 0.1840 & 0.3205 & 0.4715 & 0.4767 & 0.5707 & 0.5170 \\
\hline $\bar{x}_{21}(0)$ & $\bar{x}_{22}(0)$ & $\bar{x}_{23}(0)$ & $\bar{x}_{24}(0)$ & $\bar{x}_{25}(0)$ & $\bar{x}_{26}(0)$ & $\bar{x}_{27}(0)$ & $\bar{x}_{28}(0)$ & $\bar{x}_{29}(0)$ & $\bar{x}_{30}(0)$ \\
\hline 0.4432 & 0.5619 & 0.4958 & 0.3558 & 0.5019 & 0.4959 & 0.98 & 0.5758 & 0.5715 & 0.5587 \\
\hline
\end{tabular}

The obtained optimal solutions are listed in Table 4 . Then, based on the HK model, the adjusted initial opinions of these 30 workers will be evolved to reach a consensus at time $t^{*}=5$, as depicted in Fig. 7 .

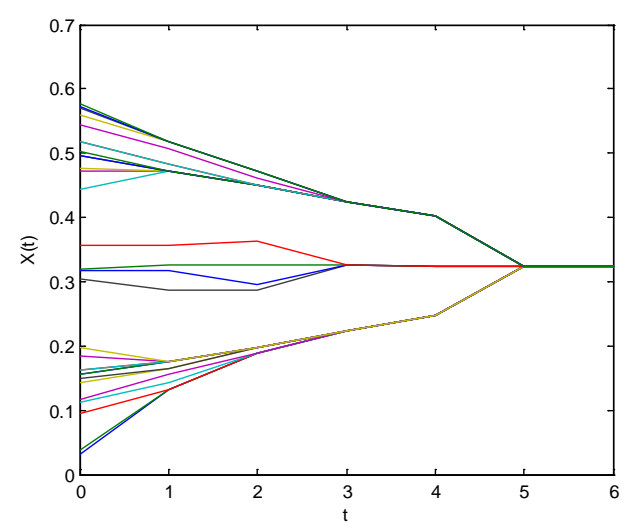

Fig. 7. The evolution of the optimal opinions under Case II

\section{B. Simulation analysis}

In this subsection, a comparative study on the performance of the MAT model is carried out. To do so, another consensus model (we refer to the GMAT model) is firstly developed. Afterwards, the simulation experiment will be carried out for both MAT and GMAT models with the same initial opinions and time constraints.

\section{B1. The GMAT model}

In the GMAT model, the opinions of individuals at each time $t\left(t=0,1, \ldots, t^{*}\right)$ may be adjusted, and then they will evolve following the HK model.

Before presenting the GMAT model, we define some related notations:

Let $\overline{\bar{x}}_{i}(t)$ be the adjusted opinion of individual $A_{i}$ at time $t$ in the GMAT model. Let $y_{i}(t+1)$ be the opinion that $\overline{\bar{x}}_{i}(t)$ will evolve to at time $t+1$, for $t=$ $0,1, \ldots, t^{*}-1$, and $y_{i}(0)=x_{i}(0)$. Thus, $y_{i}(t+1)$ is determined by

$y_{i}(t+1)=\frac{\sum_{j=1}^{N} e_{i j}^{(t)} \overline{\bar{x}}_{i}(t)}{\sum_{j=1}^{N} e_{i j}^{(t)}}, t=0,1, \ldots, t^{*}-1, i=1,2, \ldots, N$

where $e_{i j}^{(t)}=\left\{\begin{array}{l}1,\left|\overline{\bar{x}}_{i}(t)-\overline{\bar{x}}_{j}(t)\right| \leq \varepsilon \\ 0, \text { otherwise }\end{array}\right.$

Then, the opinions of individuals in the GMAT model should have the minimum adjustments, i.e.,

$$
\operatorname{Min} \sum_{t=0}^{t^{*}} \sum_{i=1}^{N}\left|\overline{\bar{x}}_{i}(t)-y_{i}(t)\right|
$$

Meanwhile, the adjusted opinions of individuals in GMAT model should reach a consensus at time $t^{*}$, i.e.,

$$
\overline{\bar{x}}_{i}\left(t^{*}\right)=\overline{\bar{x}}_{j}\left(t^{*}\right), \quad i, j=1,2, \ldots, N
$$

$$
\left\{\begin{array}{c}
\operatorname{Min} \sum_{t=0}^{t^{*}} \sum_{i=1}^{N}\left|\overline{\bar{x}}_{i}(t)-y_{i}(t)\right| \\
y_{i}(t+1)=\frac{\sum_{j=1}^{N} e_{i j}^{(t)} \overline{\bar{x}}_{i}(t)}{\sum_{j=1}^{N} e_{i j}^{(t)}} \\
y_{i}(0)=x_{i}(0) \\
\overline{\bar{x}}_{i}\left(t^{*}\right)=\overline{\bar{x}}_{j}\left(t^{*}\right) \\
e_{i j}^{(t)}=\left\{\begin{array}{l}
1,\left|\overline{\bar{x}}_{i}(t)-\overline{\bar{x}}_{j}(t)\right| \leq \varepsilon \\
0, \text { otherwise }
\end{array}\right. \\
\overline{\bar{x}}_{i}(t) \in[0,1]
\end{array}\right.
$$

where $\overline{\bar{x}}_{i}(t) \quad\left(i=1,2, \ldots, N\right.$ and $\left.t=0,1, \ldots, t^{*}\right)$ are decision variables.

Clearly, when $t^{*}=0$, the GMAT model can be equivalently transformed as the MAT model.

Note 2. Both the MAT and GMAT model can assist the individuals to reach consensus in a required time. In the former model, only the initial opinions of individuals are adjusted. Meanwhile in the latter model, the opinions of individuals at each time $t\left(t=0,1, \ldots, t^{*}\right)$ are adjusted.

To solve the GMAT model, we propose the PSO algorithm II is provided in Appendix $\mathrm{H}$.

B2. Simulation experiment

In this subsection, we design a simulation experiment to compare the MAT model with the GMAT model. Let $z_{1}^{*}$ and $z_{2}^{*}$ be the objective function values of the MAT model and the GMAT model, respectively, where $z_{1}^{*}=$ $\sum_{i=1}^{N}\left|\bar{x}_{i}^{*}(0)-x_{i}(0)\right|$ and $z_{2}^{*}=\sum_{t=0}^{t^{*}} \sum_{i=1}^{N}\left|\overline{\bar{x}}_{i}(t)-y_{i}(t)\right|$.

Then, we propose a simulation method to calculate the values of $z_{1}^{*}$ and $z_{2}^{*}$ under different $t^{*}$ values.

\section{Simulation method I}

Input: $t^{*}$ and $\varepsilon$.

Output: $z_{1}^{*}$ and $z_{2}^{*}$.

Step 1. The initial opinions $X(0)$ of individuals are the same as those from Tables 1 or 3 .

Step 2. The MAT model and the GMAT model are constructed, respectively. Then, use the PSO algorithm I and the PSO algorithm II to solve the MAT model and GMAT model, respectively.

Step 3. Obtain the optimal solutions of the MAT model and the GMAT model, and calculate the values of $z_{1}^{*}$ and $z_{2}^{*}$.

Step 4. Output the value of $z_{1}^{*}$ and $z_{2}^{*}$.

Note 2. When using the PSO algorithm I and the PSO algorithm II, we set the same parameters Subsection V-A.

Let $\varepsilon=0.2$. Based on the initial opinions given in Tables 1 and 3 , we set different $t^{*}$ values, and run the simulation method I 1000 times to calculate the average values of $z_{1}^{*}$ and $z_{2}^{*}$, which are shown in Figs. 8-9.

The GMAT model is thus formulated as follows: 


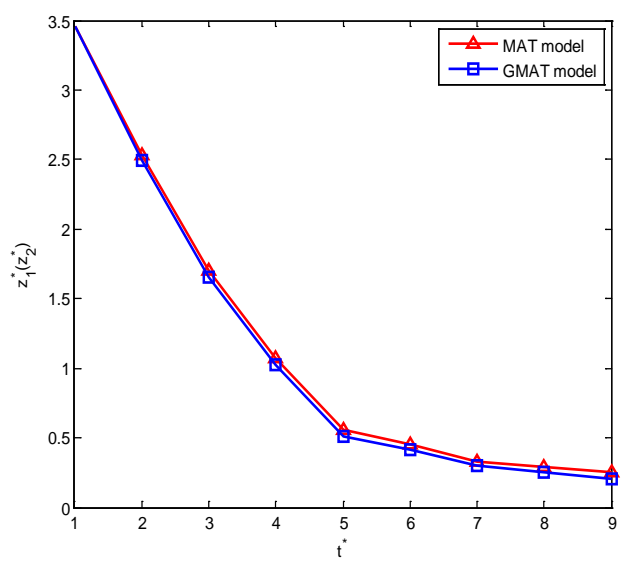

Fig. 8. The average values of $z_{1}^{*}$ and $z_{2}^{*}$ under different $t^{*}$ values based on the opinions in Table 1 .

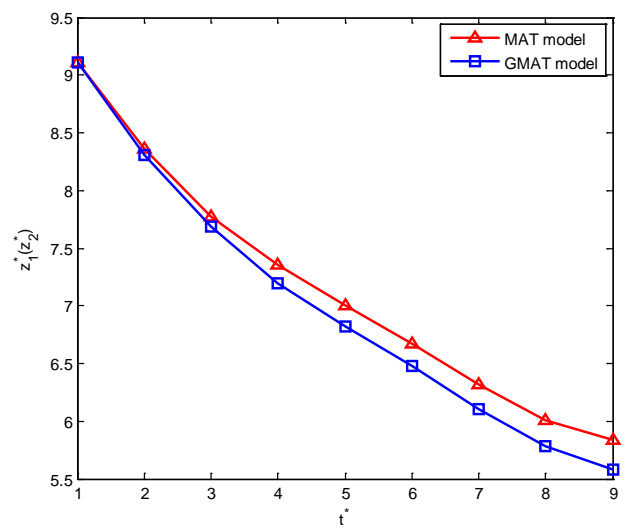

Fig. 9. The average values of $z_{1}^{*}$ and $z_{2}^{*}$ under different $t^{*}$ values based on the opinions in Table 3 .

From Figs. 8-9, we notice the following observations:

(i) Both the values of $z_{1}^{*}$ and $z_{2}^{*}$ decrease as $t^{*}$ increases. This implies that the opinions of individuals in both MAT and GMAT models are subjected to higher adjustments when the time requested for reaching consensus decreases. (ii) There is a small difference between the values of $z_{1}^{*}$ and $z_{2}^{*}$. This implies that: the amounts of adjustments in MAT model are closer aligned to those in the GMAT model. Thus, the performances of the MAT model and the GMAT model are very similar.

Furthermore, based on simulation experiments, we summarize the advantages of the MAT model over the GMAT model as follows:

(i) From Fig. 4, we observed that: the amounts of adjustments in the MAT model are approximate to those in the GMAT model. This implies that the performances of the MAT model and the GMAT model are very similar.

(ii) Since the MAT model is considered as a special case of GMAT model, thus the computation complexity of the GMAT model is higher than that of the MAT model.

(iii) In the simulation experiment, we observe some individuals in the GMAT model may adjust their opinions at different time. While in the MAT model, each individual adjust his/her opinion at most one time.

(iv) In the simulation experiment, we observe that the number of individuals adjusting their opinions in the GMAT model is more than that in the MAT model. This implies the number of individuals without adjustments in the MAT model is more than that in the GMAT model.

Therefore, the MAT model is considered the basic model to assist the individuals reach a consensus in a required time

\section{Comparison analysis}

In this subsection, we compare the MAT model with existing consensus reaching methods from five aspects: context, feedback adjustment, time constraint, opinion evolution and consensus result.

The detailed comparisons between the existing consensus reaching methods over the MAT model are described in Table 5.

Table 5. Comparison of the MAT model and the existing consensus reaching methods

\begin{tabular}{c|c|c|c|c|c}
\hline Method & Context & $\begin{array}{c}\text { Feedback } \\
\text { adjustments }\end{array}$ & $\begin{array}{c}\text { Time } \\
\text { constraints }\end{array}$ & $\begin{array}{c}\text { Opinion } \\
\text { evolution }\end{array}$ & $\begin{array}{c}\text { Consensus } \\
\text { result }\end{array}$ \\
\hline Dabarera et al., [21] & OD & $\begin{array}{c}\text { Not } \\
\text { considered }\end{array}$ & $\begin{array}{c}\text { Not } \\
\text { considered }\end{array}$ & Considered & $\begin{array}{c}\text { Consensus or } \\
\text { fragmentation }\end{array}$ \\
\hline Ding et al., [22] & OD & $\begin{array}{c}\text { No minimum } \\
\text { adjustment }\end{array}$ & $\begin{array}{c}\text { Not } \\
\text { considered }\end{array}$ & Considered & $\begin{array}{c}\text { Unique } \\
\text { opinion }\end{array}$ \\
\hline Hegselmann et al., [23] & OD & $\begin{array}{c}\text { No minimum } \\
\text { adjustment }\end{array}$ & $\begin{array}{c}\text { Not } \\
\text { considered }\end{array}$ & Considered & $\begin{array}{c}\text { Unique } \\
\text { opinion }\end{array}$ \\
\hline Liu et al., [29] & GDM & $\begin{array}{c}\text { No minimum } \\
\text { adjustment }\end{array}$ & $\begin{array}{c}\text { Not } \\
\text { considered }\end{array}$ & $\begin{array}{c}\text { Not } \\
\text { considered }\end{array}$ & $\begin{array}{c}\text { Soft } \\
\text { consensus }\end{array}$ \\
\hline Wu et al., [30] & GDM & $\begin{array}{c}\text { No minimum } \\
\text { adjustment }\end{array}$ & $\begin{array}{c}\text { Not } \\
\text { considered }\end{array}$ & $\begin{array}{c}\text { Not } \\
\text { considered }\end{array}$ & $\begin{array}{c}\text { Soft } \\
\text { consensus }\end{array}$ \\
\hline Gong et al., [37] & GDM & $\begin{array}{c}\text { Minimum } \\
\text { adjustment }\end{array}$ & $\begin{array}{c}\text { Not } \\
\text { considered }\end{array}$ & $\begin{array}{c}\text { Not } \\
\text { considered }\end{array}$ & $\begin{array}{c}\text { Soft } \\
\text { consensus }\end{array}$ \\
\hline The MAT model & OD & $\begin{array}{c}\text { Minimum } \\
\text { adjustment }\end{array}$ & Considered & Considered & $\begin{array}{c}\text { Unique } \\
\text { opinion }\end{array}$ \\
\hline
\end{tabular}

reached in a required time.

From Table 5, we obtain the following observations:

(i) In the existing methods, the time constraints are not considered. This implies that the existing consensus reaching method can't guarantee that the consensus can be (ii) In the existing methods in OD context, the minimum adjustments are not considered. This implies that the existing methods in OD context will take the large consensus cost to reach a consensus. 
(iii) In the existing methods in GDM context, the opinion evolutions are not considered, which contradicts the fact that some individuals often share and evolve their opinions and preferences through the interactions.

(iv) The time constraints, the minimum adjustments and opinion evolutions are considered in the MAT model. This implies that the proposed MAT model has the distinct advantages over the existing methods.

\section{CONCLUSION}

In this contribution, we propose a new consensus model that takes into consideration the time constraints and the minimum adjustments in a group of individuals with bounded confidence. The main contributions of this research work are as follows:

(1) The MAT model presents minimum adjustments rules to modify the initial opinions of individuals, which can further influence the opinion evolutions of individuals to reach consensus in a required time.

(2)The equivalent forms of the MAT model in different time constraints are provided. If the required time is not high the MAT model can be easily solved using an optimization software package. On the contrary, when the required time become higher, we propose the PSO I algorithm to solve the MAT model.

(3) Properties of the MAT model are discussed. These properties prove that: i) the shorter the required time the larger adjustments in the initial opinions are necessary in the initial opinions in order to reach a consensus, ii) the original opinions' sequence consistency will be maintained after modifying the initial opinions, iii) the average opinion will have the minimum adjustment in the case of uniform initial opinions, and iv) some special cases when all the opinions of individuals will evolve to reach a consensus without modifying their initial opinions.

(4) A numerical example is given to show the usefulness of the MAT model. A comparison with another consensus model (the GMAT model) is then conducted via simulation experiments. The results obtained show that the performances of the MAT model and the GMAT model are similar, although the MAT model presents lower computational more efficient.

In this paper it is assumed that individuals express their preferences honestly. However, in real-life consensus reaching process, individuals often hide their true preferences, and express dishonest preferences to exhibit the strategic manipulation behaviours [39]. Meanwhile, in this paper, the robustness of the MAT model is not studied. However, the robustness of the MAT model always has great influence on the quality of consensus reaching. Therefore, in the future, we will investigate the bounded confidence consensus reaching considering the strategic manipulation behaviours, and investigate the robustness of the MAT model through the simulation experiments with both real and random generated data.

\section{APPENDIX}

\section{A. The proof of Theorem 3.}

Proof. For any two individuals $A_{i}$ and $A_{j}$, when $\left|\bar{x}_{i}(1)-\bar{x}_{j}(1)\right| \leq \varepsilon$, then $A_{i}$ and $A_{j}$ will have the same confidence set at time $t=1$, i.e., $I\left(A_{i}, X(1)\right)=$
$I\left(A_{j}, X(1)\right)$. Furthermore, all the individuals will reach a consensus at time $t=2$, i.e., $\bar{x}_{i}(2)=\bar{x}_{j}(2)$, for $i, j=1,2, \cdots, N$.

Meanwhile, when $r_{i j}^{(0)}=1$, then we obtain:

$$
\left\{\begin{array}{l}
\bar{x}_{j}(0)-\bar{x}_{i}(0) \geq-\varepsilon, \\
\bar{x}_{j}(0)-\bar{x}_{i}(0) \leq \varepsilon, \\
\bar{x}_{j}(0)-\bar{x}_{i}(0)<-\varepsilon+M=M, \\
\bar{x}_{j}(0)-\bar{x}_{i}(0)>\varepsilon-M=-M .
\end{array}\right.
$$

When $r_{i j}^{(0)}=0$, since $y_{i j}^{(0)}-r_{i j}^{(0)} \geq 0$ and $y_{i j}^{(0)}+$ $r_{i j}^{(0)}<2$, then we consider two cases: (i) $r_{i j}^{(0)}=0$ and $y_{i j}^{(0)}=0$, and (ii) $r_{i j}^{(0)}=0$ and $y_{i j}^{(0)}=1$.

In case (i), we obtain:

$$
\left\{\begin{array}{l}
\bar{x}_{j}(0)-\bar{x}_{i}(0) \geq-\varepsilon-M=-M, \\
\bar{x}_{j}(0)-\bar{x}_{i}(0) \leq \varepsilon+M=M, \\
\bar{x}_{j}(0)-\bar{x}_{i}(0)<-\varepsilon, \\
\bar{x}_{j}(0)-\bar{x}_{i}(0)>\varepsilon-M=-M .
\end{array}\right.
$$

In case (ii), we obtain:

$$
\left\{\begin{array}{l}
\bar{x}_{j}(0)-\bar{x}_{i}(0) \geq-\varepsilon-M=-M, \\
\bar{x}_{j}(0)-\bar{x}_{i}(0) \leq \varepsilon+M=M, \\
\bar{x}_{j}(0)-\bar{x}_{i}(0)<-\varepsilon+M=M, \\
\bar{x}_{j}(0)-\bar{x}_{i}(0)>\varepsilon .
\end{array}\right.
$$

Obviously, we obtain $-\varepsilon \leq \bar{x}_{j}(0)-\bar{x}_{i}(0) \leq \varepsilon$ when $r_{i j}^{(0)}=1$. And we obtain $\bar{x}_{j}(0)-\bar{x}_{i}(0)<-\varepsilon$ and $\bar{x}_{j}(0)-\bar{x}_{i}(0)>\varepsilon$ are the mutually exclusive constraints. Thus, it is $\bar{x}_{j}(0)-\bar{x}_{i}(0)<-\varepsilon$ or $\bar{x}_{j}(0)-\bar{x}_{i}(0)>\varepsilon$ when $r_{i j}^{(0)}=0$.

This completes the proof of Theorem 3.

\section{B. The proof of Theorem 4.}

Proof. For any two individuals $A_{\sigma(i)}$ and $A_{\sigma(i+1)}$, if $\left|\bar{x}_{\sigma(i)}(0)-\bar{x}_{\sigma(i+1)}(0)\right|>\varepsilon$, then we have: $I\left(A_{\sigma(i)}, \bar{X}(0)\right)$ $\cap I\left(A_{\sigma(i+1)}, \bar{X}(0)\right)=\emptyset$, for $i=1,2, \cdots, N-1$.

$$
\text { Since } \bar{x}_{\sigma(i)}(1)=\frac{\sum_{A_{j} \in I\left(A_{\sigma(i)}, \bar{X}(0)\right)} \bar{x}_{j}(0)}{\# I\left(A_{\sigma(i)}, \bar{X}(0)\right)} \text { and } \bar{x}_{\sigma(i+1)}(1)
$$

$=\frac{\sum_{A_{j} \in I\left(A_{\sigma(i+1)}, \bar{X}(0)\right)} \bar{x}_{j}(0)}{\# I\left(A_{\sigma(i+1)}, \bar{X}(0)\right)}$, we obtain: $\bar{x}_{\sigma(i)}(1) \leq \bar{x}_{\sigma(i)}(0)$ and $\bar{x}_{\sigma(i+1)}(1) \geq \bar{x}_{\sigma(i+1)}(0)$.

Furthermore, we obtain: $\bar{x}_{\sigma(i+1)}(1)-\bar{x}_{\sigma(i)}(1) \geq$ $\bar{x}_{\sigma(i+1)}(0)-\bar{x}_{\sigma(i)}(0) \geq \varepsilon$. By repeating the above operation, we can prove:

$$
\begin{aligned}
\bar{x}_{\sigma(i+1)}\left(t^{*}\right)-\bar{x}_{\sigma(i)}\left(t^{*}\right) & \geq \bar{x}_{\sigma(i+1)}\left(t^{*}-1\right)-\bar{x}_{\sigma(i)}\left(t^{*}-1\right) \\
& \geq \cdots \geq \bar{x}_{\sigma(i+1)}(0)-\bar{x}_{\sigma(i)}(0) \geq \varepsilon .
\end{aligned}
$$

Obviously, consensus among the opinions of individuals will not be reached under the condition $\left|\bar{x}_{\sigma(i)}(0)-\bar{x}_{\sigma(i+1)}(0)\right|>\varepsilon$. Then, $\left|\bar{x}_{\sigma(i)}(0)-\bar{x}_{\sigma(i+1)}(0)\right|$ $\leq \varepsilon$, for $i=1,2, \cdots, N-1$, is the necessary conditions for reaching a consensus.

This completes the proof of Theorem 4 .

\section{The proof of Property 1.}

Proof. Let $X^{t_{1}}(0)=\left(x_{1}^{t_{1}}(0), x_{2}^{t_{1}}(0), \ldots, x_{N}^{t_{1}}(0)\right)$ and $X^{t_{2}}(0)=\left(x_{1}^{t_{2}}(0), x_{2}^{t_{2}}(0), \ldots, x_{N}^{t_{2}}(0)\right)$ be the optimal solutions of model (7) when $t^{*}=t_{1}$ and $t^{*}=t_{2}$. Obviously, we obtain: $A D\left(t_{1}\right)=\sum_{i=1}^{N}\left|x_{i}(0)-x_{i}^{t_{1}}(0)\right|$ 
and $A D\left(t_{2}\right)=\sum_{i=1}^{N}\left|x_{i}(0)-x_{i}^{t_{2}}(0)\right|$.

When $t^{*}=t_{1}$, model (10) can be transformed as follows:

$$
\left\{\begin{array}{l}
\operatorname{Min} \sum_{i=1}^{N}\left|\bar{x}_{i}(0)-x_{i}(0)\right| \\
\bar{x}_{i}(t+1)=\frac{\sum_{j=1}^{N} r_{i j}^{(t)} \bar{x}_{j}(t)}{\sum_{j=1}^{N} r_{i j}^{(t)}} \\
\bar{x}_{i}\left(t_{1}\right)=\bar{x}_{j}\left(t_{1}\right) \\
r_{i j}^{(t)}= \begin{cases}1, & \bar{x}_{i}(t)-\bar{x}_{j}(t) \mid \leq \varepsilon \\
0, & \text { otherwise }\end{cases} \\
\bar{x}_{i}(0) \in[0,1]
\end{array}\right.
$$

And when $t^{*}=t_{2}$, model (7) can be transformed as follows:

$$
\left\{\begin{array}{l}
\operatorname{Min} \sum_{i=1}^{N}\left|\bar{x}_{i}(0)-x_{i}(0)\right| \\
\bar{x}_{i}(t+1)=\frac{\sum_{j=1}^{N} r_{i j}^{(t)} \bar{x}_{j}(t)}{\sum_{j=1}^{N} r_{i j}^{(t)}} \\
\bar{x}_{i}\left(t_{2}\right)=\bar{x}_{j}\left(t_{2}\right) \\
r_{i j}^{(t)}= \begin{cases}1, & \bar{x}_{i}(t)-\bar{x}_{j}(t) \mid \leq \varepsilon \\
0, & \text { otherwise }\end{cases} \\
\bar{x}_{i}(0) \in[0,1]
\end{array}\right.
$$

If the consensus among the individuals is reached at time $t$, then the consensus among the individuals will be maintained. Thus, if $t_{1}<t_{2}$, then we obtain: $X^{t_{1}}(0)=$ $\left(x_{1}^{t_{1}}(0), x_{2}^{t_{1}}(0), \ldots, x_{N}^{t_{1}}(0)\right)$ is a feasible solution of model (18). Obviously, we obtain: $A D\left(t_{1}\right)<A D\left(t_{2}\right)$.

This completes the proof of Property 1.

D. The proof of Property 2.

Proof. Let $x_{\sigma(i)}(0)$ be the $i$ th smallest value in the set $\left\{x_{1}(0), x_{2}(0), \ldots, x_{2}(0)\right\}$. Assume that $x_{\sigma(i)}(0) \leq$ $x_{\sigma(j)}(0)$ but $\bar{x}_{\sigma(i)}(0) \geq \bar{x}_{\sigma(j)}(0)$. Meanwhile, we assume that $x_{\sigma(k)}(0) \leq x_{\sigma(l)}(0)$ but $\bar{x}_{\sigma(k)}(0) \leq \bar{x}_{\sigma(l)}(0)$, for $i<k, l<j$.

Then, we obtain:

$$
\begin{gathered}
\sum_{q=i}^{l}\left|x_{\sigma(q)}(0)-\bar{x}_{\sigma(q)}(0)\right|-\sum_{q=l+1}^{j-1}\left|x_{\sigma(q)}(0)-\bar{x}_{\sigma(q)}(0)\right| \\
\quad-\left|x_{\sigma(i)}(0)-\bar{x}_{\sigma(j)}(0)\right|-\left|x_{\sigma(j)}(0)-\bar{x}_{\sigma(i)}(0)\right| \\
=\left|x_{\sigma(i)}(0)-\bar{x}_{\sigma(i)}(0)\right|-\left|x_{\sigma(j)}(0)-\bar{x}_{\sigma(j)}(0)\right|
\end{gathered}
$$$$
-\left|x_{\sigma(i)}(0)-\bar{x}_{\sigma(j)}(0)\right|-\left|x_{\sigma(j)}(0)-\bar{x}_{\sigma(i)}(0)\right|
$$

Since $x_{\sigma(i)}(0) \leq x_{\sigma(j)}(0)$ and $\bar{x}_{\sigma(i)}(0) \geq \bar{x}_{\sigma(j)}(0)$, we obtain:

$$
\begin{aligned}
& \left|x_{\sigma(i)}(0)-\bar{x}_{\sigma(j)}(0)\right|+\left|x_{\sigma(j)}(0)-\bar{x}_{\sigma(i)}(0)\right| \\
\leq & \left|x_{\sigma(i)}(0)-\bar{x}_{\sigma(i)}(0)\right|+\left|x_{\sigma(j)}(0)-\bar{x}_{\sigma(j)}(0)\right| .
\end{aligned}
$$

Then, we obtain:

$\sum_{q=i}^{l}\left|x_{\sigma(q)}(0)-\bar{x}_{\sigma(q)}(0)\right|-\sum_{q=l+1}^{j-1}\left|x_{\sigma(q)}(0)-\bar{x}_{\sigma(q)}(0)\right|$ $-\left|x_{\sigma(i)}(0)-\bar{x}_{\sigma(j)}(0)\right|-\left|x_{\sigma(j)}(0)-\bar{x}_{\sigma(i)}(0)\right| \geq 0$

Obviously, this

contradicts that $\left(\bar{x}_{\sigma(i)}(0), \bar{x}_{\sigma(i+1)}(0), \ldots, \bar{x}_{\sigma(j)}(0)\right)$ belongs to the optimal solution.

This completes the proof of Property 2.

\section{E. The proof of Property 3.}

Proof. We consider the extreme case: $x_{i+1}(0)-$ $x_{i}(0)=\varepsilon$, for $N=1,2,3$. Then, based on the HK model, we obtain: $\quad x_{1}(1)=x_{1}(0)+\frac{\varepsilon}{2}, \quad x_{2}(1)=x_{1}(0)+\varepsilon$, $x_{3}(1)=x_{1}(0)+2 \varepsilon$ and $x_{4}(1)=x_{1}(0)+\frac{3 \varepsilon}{2}$.

Similarly, we obtain:

$$
x_{1}(2)=x_{1}(0)+\frac{3 \varepsilon}{4}, x_{2}(2)=x_{1}(0)+\frac{7 \varepsilon}{6},
$$

$$
\begin{aligned}
& x_{3}(2)=x_{1}(0)+\frac{11 \varepsilon}{6}, x_{4}(2)=x_{1}(0)+\frac{9 \varepsilon}{4} . \\
& x_{1}(3)=x_{1}(0)+\frac{23 \varepsilon}{24}, x_{2}(3)=x_{1}(0)+\frac{5 \varepsilon}{4} \\
& x_{3}(3)=x_{1}(0)+\frac{7 \varepsilon}{4}, x_{4}(3)=x_{1}(0)+\frac{29 \varepsilon}{24} . \\
& x_{1}(4)=x_{1}(0)+\frac{95 \varepsilon}{72}, x_{2}(4)=x_{1}(0)+\frac{3 \varepsilon}{2} \\
& x_{3}(4)=x_{1}(0)+\frac{3 \varepsilon}{2}, x_{4}(4)=x_{1}(0)+\frac{121 \varepsilon}{72} . \\
& x_{1}(5)=x_{1}(0)+\frac{3 \varepsilon}{2}, x_{2}(4)=x_{1}(0)+\frac{3 \varepsilon}{2} \\
& x_{3}(4)=x_{1}(0)+\frac{3 \varepsilon}{2}, x_{4}(4)=x_{1}(0)+\frac{3 \varepsilon}{2} .
\end{aligned}
$$

Clearly, in this extreme case, the opinions of individuals reach consensus at time $t^{*}=5$.

This completes the proof of Property 3.

\section{F. The proof of Property 4.}

Proof. Since $x_{\sigma(2)}(0)-x_{\sigma(1)}(0) \leq \varepsilon$ and $x_{\sigma(N)}(0)$ $-x_{\sigma(N-1)}(0) \leq \varepsilon$, we obtain: $A_{\sigma(2)} \in I\left(A_{\sigma(1)}, X(0)\right)$ and $A_{\sigma(N-1)} \in I\left(A_{\sigma(N)}, X(0)\right)$.

Based on the HK model, we obtain:

$$
x_{\sigma(1)}(1)=\frac{\sum_{A_{j} \in I\left(A_{\sigma(1)}, X(0)\right)} x_{j}(0)}{\# I\left(A_{\sigma(1)}, X(0)\right)}
$$

and

$$
x_{\sigma(N)}(1)=\frac{\sum_{A_{j} \in I\left(A_{\sigma(N)}, X(0)\right)} x_{j}(0)}{\# I\left(A_{\sigma(N)}, X(0)\right)}
$$

Let $l_{1}=\# I\left(A_{\sigma(1)}, X(0)\right)$ and $l_{2}=\# I\left(A_{\sigma(N)}, X(0)\right)$, where $l_{1}, l_{2} \geq 2$. Then we obtain:

and

$$
\begin{gathered}
x_{\sigma(1)}(1)=\frac{\sum_{A_{j} \in I\left(A_{\sigma(1)}, X(0)\right)} x_{j}(0)}{l_{1}} \\
\geq \frac{x_{\sigma(1)}(0)+\left(l_{1}-1\right) x_{\sigma(2)}(0)}{l_{1}} \\
\geq \frac{x_{\sigma(1)}(0)+x_{\sigma(2)}(0)}{2} .
\end{gathered}
$$

$$
\begin{aligned}
x_{\sigma(N)}(1) & =\frac{\sum_{A_{j} \in I}\left(A_{\sigma(1)}, X(0)\right)^{x_{j}(0)}}{l_{2}} \\
& \leq \frac{x_{\sigma(N)}(0)+\left(l_{2}-1\right) x_{\sigma(N-1)}(0)}{l_{2}} \\
& \leq \frac{x_{\sigma(N)}(0)+x_{\sigma(N-1)}(0)}{2} .
\end{aligned}
$$

$x_{\sigma(N)}(1)-x_{\sigma(1)}(1) \leq \frac{x_{\sigma(N)}(0)+x_{\sigma(N-1)}(0)}{2}-\frac{x_{\sigma(1)}(0)+x_{\sigma(2)}(0)}{2}$ $\leq \varepsilon$.

Furthermore, we obtain: $I\left(A_{\sigma(i)}, X(1)\right)=A$, for $i=1,2, \ldots, N$. Thus, consensus among the individuals will be reached at time $t^{*}=2$.

This completes the proof of Property 4.

G. The proof of Property 5.

Proof. Since $\left|x_{\sigma(1)}(0)-x_{k}(0)\right| \leq \varepsilon$ and $\mid x_{\sigma(N)}(0)$ $-x_{k}(0) \mid \leq \varepsilon$, then we obtain: $A_{k} \in I\left(A_{i}, X(0)\right)$, for $i=1$, $2, \ldots, N$.

According to the HK model, we have $x_{k}(1)=$ $\sum_{i=1}^{N} \frac{x_{i}(0)}{N}$.

Without loss of generality, we assume that $x_{k}(1) \leq$ $x_{k}(0)$. Let $d_{1}=x_{k}(0)-x_{k}(1)$. Since $\mid x_{\sigma(N)}(1)-$ $x_{k}(1)|\leq| x_{\sigma(N)}(0)-x_{k}(0) \mid$, we have

$$
\begin{aligned}
\left|x_{\sigma(N)}(1)-x_{k}(1)\right| & =x_{\sigma(N)}(1)-x_{k}(0)+d_{1} \\
& \leq x_{\sigma(N)}(0)-x_{k}(0)
\end{aligned}
$$

Then, we obtain $x_{\sigma(N)}(0)-x_{\sigma(N)}(1) \geq d_{1}$. Also because $\left|x_{\sigma(N)}(1)-x_{k}(1)\right| \leq\left|x_{\sigma(N)}(0)-x_{k}(0)\right|$, we 
obtain: $x_{k}(2)=\sum_{i=1}^{N} \frac{x_{i}(1)}{N}$. Since $x_{k}(1) \leq x_{k}(0)$, we can easily obtain: $x_{k}(2) \leq x_{k}(1)$.

Furthermore, we obtain: $\left|x_{\sigma(1)}(2)-x_{k}(2)\right| \leq$ $\left|x_{\sigma(1)}(1)-x_{k}(1)\right|$ and $\left|x_{\sigma(N)}(2)-x_{k}(2)\right| \leq \mid x_{\sigma(N)}(1)-$ $x_{k}(1) \mid$.

By repeating the above proof, we can prove:

$$
\begin{aligned}
\left|x_{\sigma(1)}(t)-x_{k}(t)\right| & \leq \cdots \leq\left|x_{\sigma(1)}(2)-x_{k}(2)\right| \\
& \leq\left|x_{\sigma(1)}(1)-x_{k}(1)\right|
\end{aligned}
$$

and

$$
\begin{aligned}
\left|x_{\sigma(N)}(t)-x_{k}(t)\right| & \leq \cdots \leq\left|x_{\sigma(N)}(2)-x_{k}(2)\right| \\
& \leq\left|x_{\sigma(N)}(1)-x_{k}(1)\right|
\end{aligned}
$$

Obviously, the values of $\left|x_{\sigma(N)}(t)-x_{k}(t)\right|$ and $\left|x_{\sigma(N)}(t)-x_{k}(t)\right|$ converge to 0 . As a result, consensus among the individuals will be obtained.

This completes the proof of Property 5.

\section{H. The PSO algorithm II.}

Following the general framework of classical PSO algorithm, the key steps of PSO algorithm II are described as follows:

\section{(1) Initiation}

Let $P=\left\{P_{1}, P_{2}, \ldots, P_{N L}\right\} \quad$ and $\quad X_{q}^{l}(0)=\left(x_{q 1}^{l}(0)\right.$, $\left.x_{q 2}^{l}(0), \ldots, x_{q N}^{l}(0)\right)$ be as defined before. Without loss of generality, assume that $x_{1}(0) \leq x_{2}(0) \leq \cdots \leq x_{N}(0)$. For two opinions $x_{i}(0)$ and $x_{i+1}(0)$, if $x_{i+1}(0)-x_{i}(0) \leq \varepsilon$, then we regard that individuals $A_{i}$ and $A_{i+1}$ belong to one cluster. Then, the individuals will be divided into $e$ clusters $s_{1}, s_{2}, \ldots, s_{e}$. We use $\# s_{1}, \# s_{2}, \ldots, \# s_{e}$ to denote their cardinalities.

Assume that $s_{y}=\left\{A_{y_{1}}, A_{y_{1}+1}, \ldots, A_{y_{1}+\# s_{y}-1}\right\}$, where $\# s_{y}=\max \left\{\# s_{1}, \# s_{2}, \ldots, \# s_{e}\right\}$ and $y \leq e$. Then, we have: $x_{q h}^{1}(0)=x_{h}(0), h=y_{1}, \ldots, y_{1}+\# s_{y}-1$ (24a) $x_{q(h-1)}^{1}(0)=x_{q h}^{1}(0)-\varepsilon \times \operatorname{rand}(1), h=2, \ldots, y_{1}(24 \mathrm{~b})$ $x_{q(h+1)}^{1}(0)=x_{q h}^{1}(0)+\varepsilon \times \operatorname{rand}(1), \quad h=y_{1}+$ $\# s_{y}-1, \ldots, N-1 \quad$ (24c)

Let $V_{q}^{l}(t)=\left(v_{q 1}^{l}(t), v_{q 2}^{l}(t), \ldots, v_{q N}^{l}(t)\right)$ be the velocity vector of particle $P_{q}$ at time $t$ of the $l$ th iteration, where $v_{q h}^{l}(t)$ denotes the $h$ th dimension velocity of particle $P_{q}$, and $v_{q h}^{l}(t)$ is a random number selected from $[0, \varepsilon]$. And let $V_{q}^{l}=\left(V_{q}^{l}(0), V_{q}^{l}(1), \ldots, V_{q}^{l}\left(t^{*}\right)\right)$.

\section{(2) Fitness function}

Let $Y_{q}^{l}(t+1)=\left(y_{q 1}^{l}(t+1), y_{q 2}^{l}(t+1), \ldots, y_{q N}^{l}(t+\right.$ 1)) be the opinion profile derived from $X_{q}^{l}(t)$ and $Y_{q}^{l}(0)=X(0)$, where $y_{q h}^{l}(t+1)$ denotes the opinion that $X_{q}^{l}(t)$ will evolve to at time $t+1$, i.e.,

$$
y_{q h}^{l}(t+1)=\frac{\sum_{A_{j} \in I\left(A_{h}, X(t)\right)} x_{q j}^{l}(t)}{\# I\left(A_{h}, X(t)\right)}, h=1,2, \ldots, N
$$

where $\quad I\left(A_{h}, X(t)\right)=\left\{A_{j}|| x_{q h}^{l}(t)-x_{q j}^{l}(t) \mid \leq \varepsilon\right\} \quad$ and $\# I\left(A_{h}, X(t)\right)$ denotes its cardinality.

For $y_{q h}^{l}(t+1)$, if $y_{q h}^{l}(t+1)-y_{q(h-1)}^{l}(t+1) \leq$ $y_{q h}^{l}(t)-y_{q(h-1)}^{l}(t)$ and $y_{q(h+1)}^{l}(t+1)-y_{q h}^{l}(t+1) \leq$ $y_{q(h+1)}^{l}(t)-y_{q h}^{l}(t)$, then we regard individuals $A_{h-1}, A_{h}$, $A_{h+1}$ belong to one cluster. Then the individuals will be divided into $u$ clusters $r_{1}, r_{2}, \ldots, r_{u}$.

Assume that $r_{q}=\left\{A_{u_{1}}, A_{u_{1}+1}, \ldots, A_{u_{1}+\# r_{q}-1}\right\}$, where $\# r_{q}=\left\{\# r_{1}, \# r_{2}, \ldots, \# r_{u}\right\}$ and $q \leq u$. Then, $x_{q h}^{l}(t+1)$ is determined by

$$
\begin{gathered}
x_{q h}^{l}(t+1)=y_{q h}^{l}(t+1), \\
h=u_{1}, \ldots, u_{1}+\# r_{q}-1 \\
x_{q(h-1)}^{l}(t+1)=x_{q h}^{l}(t+1)-\varepsilon \times \operatorname{rand}(1), \\
h=2, \ldots, u_{1} \\
x_{q(h+1)}^{l}(t+1)=x_{q h}^{l}(t+1)+\varepsilon \times \operatorname{rand}(1), \\
h=u_{1}+\# r_{q}-1, \ldots, N-1
\end{gathered}
$$

Let $f\left(X_{q}^{l}(0), X_{q}^{l}(1), \cdots, X_{q}^{l}\left(t^{*}\right)\right)$ be the fitness function of particle $P_{q}$ in the $l$ th iteration, where

$$
\begin{gathered}
f\left(X_{q}^{l}(0), X_{q}^{l}(1), \cdots, X_{q}^{l}\left(t^{*}\right)\right)= \\
\left\{\begin{array}{cl}
\sum_{t=0}^{t^{*}} \sum_{i=1}^{N}\left|x_{q i}^{l}(t)-y_{q i}^{l}(t)\right|, & y_{q 1}^{l}\left(t^{*}\right)=\cdots=y_{q N}^{l}\left(t^{*}\right) \\
M, & \text { otherwise }
\end{array}\right.
\end{gathered}
$$

\section{(3) Updating the velocity and position of particles}

Let $z_{p q}$ and $z_{g}$ be as defined before. And let $X_{q}^{l}=\left(X_{q}^{l}(0), X_{q}^{l}(1), \cdots, X_{q}^{l}\left(t^{*}\right)\right)$. Then, $z_{p q}$ and $z_{g}$ can be determined by

$$
\begin{aligned}
& z_{p q}=\arg \min _{x \in\left\{X_{q}^{1}, X_{q}^{2}, \ldots, X_{q}^{l}\right\}} f(x) \\
& z_{g}=\arg \min _{x \in\left\{z_{p 1}, z_{p 2}, \ldots, z_{p N L}\right\}} f(x)
\end{aligned}
$$

Let $l_{\max }$ be as before. When $l<l_{\max }$, the velocity and position of particles will be updated. Let $V_{q}^{l+1}=$ $\left(V_{q}^{l+1}(0), V_{q}^{l+1}(1), \cdots, V_{q}^{l+1}\left(t^{*}\right)\right)$ be the velocity vector of particle $P_{q}$ in the $l+1$ th iteration, and let $X_{q}^{l+1}=$ $\left(X_{q}^{l+1}(0), X_{q}^{l+1}(1), \cdots, X_{q}^{l+1}\left(t^{*}\right)\right)$ be the position vector of $P_{q}$ in the $l+1$ th iteration. Then, $V_{q}^{l+1}$ and $X_{q}^{l+1}$ can be determined by

$$
\begin{gathered}
V_{q}^{l+1}=w V_{q}^{l}+c_{1} r_{1}\left(z_{p q}-X_{q}^{l}\right)+c_{2} r_{2}\left(z_{g}-X_{q}^{l}\right) \\
X_{q}^{l+1}=X_{q}^{l}+V_{q}^{l+1}
\end{gathered}
$$

When $l=l_{\text {max }}$, then we output the optimal solution $z_{g}$ and the optimal fitness degree $f\left(z_{g}\right)$.

\section{REFERENCES}

[1] R. Ureña, G. Kou, Y.C. Dong, F. Chiclana, E. Herrera-Viedma, “A review on trust propagation and opinion dynamics in social networks and group decision making frameworks”, Information Sciences, vol. 478, pp. 461-475, 2018.

[2] Y.C. Dong, M. Zhan, G. Kou, Z.G. Ding, H.M. Liang, “A survey on the fusion process in opinion dynamics”, Information Fusion, vol. 43 pp. 57-65, 2018.

[3] R. Ureña, F. Chiclana, H. Fujita, E. Herrera-Viedma, “Confidence based consensus in environments with high uncertainty and incomplete information”, IOS Press, 2017.

[4] D. Guha, D. Chakraborty, "Fuzzy multi attribute group decision making method to achieve consensus under the consideration of degrees of confidence of experts' opinions”, Computers \& Industrial Engineering, vol. 60, no. 4, pp. 493-504, 2011.

[5] E. Herrera-Viedma, F.J. Cabrerizo, J. Kacprzyk, W. Pedrycz, “A review of soft consensus models in a fuzzy environment”, Information Fusion, vol. 17, pp. 4-13, 2014.

[6] I. Palomares, F.J. Estrella, L. Martínez, F. Herrera, “Consensus under a fuzzy context: Taxonomy, analysis framework AFRYCA and experimental case of study”, Information Fusion, vol. 20, pp. 252-271, 2014.

[7] Z.B. Wu, B. Jin, J.P. Xu, "Local feedback strategy for consensus building with probability-hesitant fuzzy preference relations”, Applied Soft Computing, vol. 67, pp. 691-705, 2018.

[8] Y.J. Xu, X.W. Wen, W.C. Zhang, “A two-stage consensus method for large-scale multi-attribute group decision making with an application to earthquake shelter selection”, Computers \& Industrial Engineering, vol. 116, pp. 113-129, 2018.

[9] L. Susskind, S. McKearnen, J. Thomas-Lamar, The consensus building handbook: A comprehensive guide to reaching agreement. Sage Publications, Thousand Oaks, 1999.

[10] R.A. Holley, T.M. Liggett, "Ergodic theorems for weakly interacting infinite systems and the voter model”, Annals of Probability, vol. 3, pp. 643-663, 1975.

[11]V. Sood, S. Redner, "Voter model on heterogeneous graphs”, Physical 
Review Letters, vol. 94, no. 17, 2005.

[12]K. Sznajd-Weron, J. Sznajd, "Opinion evolution in closed community", International Journal of Modern Physics C, vol. 11, no. 6, pp. 1157-1165, 2000.

[13]S. Galam, "Real space renormalization group and totalitarian paradox of majority rule voting”, Physica A, vol. 285, pp. 66-76, 2000.

[14]S. Galam, "Minority opinion spreading in random geometry", The European Physical Journal B-Condensed Matter and Complex Systems, vol. 25, no. 4, pp. 403-406, 2002.

[15]M. DeGroot, "Reaching a consensus", Journal of the American Statistical Association, vol. 69, pp. 118-121, 1974.

[16]G. Deffuant, D. Neau, F. Amblard, "Mixing beliefs among interacting agents", Advances in Complex Systems, vol. 3, no. 01n04, 2000.

[17]R. Hegselmann, U. Krause, "Opinion dynamics and bounded confidence models, analysis and simulation”, Journal of Artificial Societies \& Social Simulation, vol. 5, no. 3, 2002.

[18]A. Pluchino, V. Latora, A. Rapisarada, "Changing opinions in a changing world: A new perspective in sociophysics", International Journal of Modern Physics C, vol. 16, no. 4, pp. 515-531, 2005.

[19]A.C.R. Martins, "Continuous opinions and discrete actions in opinion dynamics problems", International Journal of Modern Physics C, vol. 19, no. 4, pp. 617-624, 2008.

[20]G. Deffuant, F. Amblard, G. Weisbuch, "Modelling group opinion shift to extreme: the smooth bounded confidence model”, arXiv preprint cond-mat/0410199, 2004.

[21]R. Dabarera, K. Premaratne, M.N. Murthi, D. Sarkar, "Consensus in the presence of multiple opinion leaders: effect of bounded confidence", IEEE Transactions on Signal and Information Processing over Networks, vol. 2, no. 3, pp. 336-349, 2016

[22]Z.G. Ding, X. Chen, Y.C. Dong, F. Herrera. "Consensus reaching in social network DeGroot Model: The roles of the Self-confidence and node degree”, Information Sciences, vol. 486, pp. 62-72, 2019.

[23]R. Hegselmann, S. König, S. Kurz, C. Niemann, J. Rambau, “Optimal opinion control: the campaign problem”, Journal of Artificial Societies \& Social Simulation, vol. 18, no. 3, 2014.

[24]X.H. Xu, Z.J. Du, X.H. Chen, C.G. Cai, “Confidence consensus-based model for large-scale group decision making: A novel approach to managing non-cooperative behaviours", Information Sciences, vol. 477, pp. 410-427, 2019.

[25]W.Q. Liu, H.J. Zhang, X. Chen, S. Yu, "Managing consensus and self-confidence in multiplicative preference relations in group decision making”, Knowledge-Based Systems, vol. 162, pp. 62-73, 2018.

[26]S. Alonso, E. Herrera-Viedma, F. Chiclana, F. Herrera, "A web based consensus support system for group decision making problems and incomplete preferences”, Information Sciences, vol. 180, no. 23, pp. 4477-4495, 2010.

[27]S. Alonso, I.J. Pérez, F.J. Cabrerizo, E. Herrera-Viedma, “A linguistic consensus model for Web 2.0 communities", Applied Soft Computing, vol. 13, no. 1, pp. 149-157, 2013.

[28]B.W. Zhang, Y.C. Dong, E. Herrera-Viedma, “Group decision making with heterogeneous preference structures: an automatic mechanism to support consensus reaching”, Group Decision and Negotiation, vol. 28 no. 3, pp. 585-617, 2019.

[29]X. Liu, Y.J. Xu, R. Montes, R. Ding, F. Herrera, “Alternative ranking-based clustering and reliability index-based consensus reaching process for hesitant fuzzy large scale group decision making”, IEEE Transactions on Fuzzy Systems, vol. 29, no. 1, pp. 159-171, 2019.

[30]Z.B. Wu, N. Ma, Z.Q. Zeng, J.P. Xu, "Integer programming models to manage consensus for uncertain MCGDM based on PSO algorithms", IEEE Transactions on Fuzzy Systems, vol. 25, no. 5, pp. 888-902, 2019.

[31]P. Gupta, C.T. Lin, M.K. Mehlawat, N. Grover, “A new method for intuitionistic fuzzy multiattribute decision making”, IEEE Transactions on Systems, Man, and Cybernetics: Systems, vol. 46, no. 9, pp. 1167-1179, 2016.

[32]D. Ben-Arieh, T. Easton, "Multi-criteria group consensus under linear cost opinion elasticity", Decision Support Systems, vol. 43, no. 3, pp. 713-721, 2007.

[33]Y.C. Dong, Y.F. Xu, H.Y. Li, B. Feng, "The OWA-based consensus operator under linguistic representation models using position indexes", European Journal of Operational Research, vol. 203, no. 2, pp. 455-463, 2010.

[34]J. Wu, L. Dai, F. Chiclana, H. Fujita, E. Herrera-Viedma, “A minimum adjustment cost feedback mechanism based consensus model for group decision making under social network with distributed linguistic trust", Information Fusion, vol. 41 pp. 232-242, 2018.

[35]D. Cheng, Z. Zhou, F. Cheng, Y. Zhou, Y. Xie, "Modeling the minimum cost consensus problem in an asymmetric costs context”, European Journal of Operational Research, vol. 270, no. 3, pp. 1122-1137, 2018.

[36]D. Ben-Arieh, T. Easton, B. Evans, "Minimum cost consensus with quadratic cost functions", IEEE Transactions on Systems, Man, and Cybernetics-Part A: Systems and Humans, vol. 39, no. 1, pp. 210-217, 2009.

[37]Z.W. Gong, H.H. Zhang, J. Forrest, L. Li, X. Xu, "Two consensus models based on the minimum cost and maximum return regarding either all individuals or one individual”, European Journal of Operational Research, vol. 240, no. 1, pp. 183-192, 2015.

[38]J. Kennedy, R.C. Eberhart, Particle swarm optimization. Proceedings-IEEE International Conference on Neural Networks, 4. IEEE Press, NJ, 1995, pp. 1942-1948.

[39]Y.T. Liu, Y.C. Dong, H.M. Liang, F. Chiclana, E. Herrera-Viedma, "Multiple attribute strategic weight manipulation with minimum cost in a group decision making context with interval attribute weights information”, IEEE Transactions on Systems, Man and Cybernetics: Systems, DOI: 10.1109/TSMC.2018.2874942 (In press)

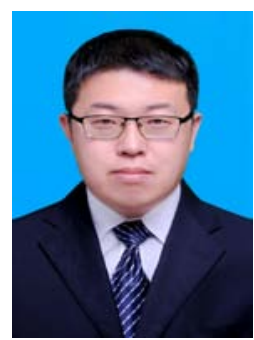

Haiming Liang is an associate research fellow at the Business School, Sichuan University, China. He received his Ph.D. degree in management from Northeastern University in 2014. His research interests include multiple attribute decision making, and opinion dynamics. Dr. Liang published several international journal papers in European Journal of Operational Research, Omega, Computers \& Industrial Engineering, Information Fusion, Journal of Artificial Societies and Social Simulation, Journal of the Operational Research Society, among others.

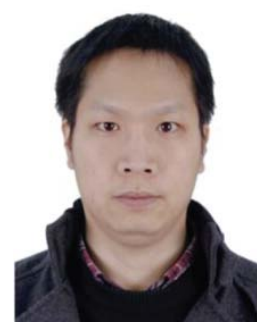

Yucheng Dong received the B.S. and M.S. degrees both in mathematics from Chongqing University, Chongqing, China, in 2002 and 2004, respectively, and the Ph.D. degree in management from Xi'an Jiaotong University, Xi'an, China, in 2008.

$\mathrm{He}$ is currently a Professor at the Business School, Sichuan University, Chengdu, China. His current research interests include consensus process, computing with words, opinion dynamics, and social network decision making. He has published more than 100 international journal papers in Decision Support Systems, European Journal of Operational Research, IEEE Transactions on Big Data, IEEE Transactions on Cybernetics, IEEE Transactions on Fuzzy Systems, IEEE Transactions on Systems, Man, and Cybernetics, Omega, among others.

Dr. Dong is a member of the editorial board of Information Fusion, an Area Editor of Computers \& Industrial Engineering, and an Associate Editor of Group Decision and Negotiation.

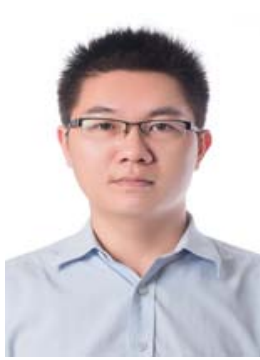

Zhaogang Ding is a lecturer at the School of Public Management, Northwest University, China. He received his Ph.D. degree in management from Sichuan University in 2017. His research interests include opinion dynamics, social network analysis and collective action. Dr. Ding published several international journal papers in Information Sciences, Journal of Artificial Societies and Social Simulation, IEEE Transactions on Big Data, among others. 


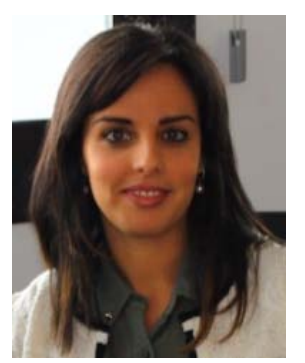

Raquel Ureña is a Senior Research Fellow in the Institute of artificial Intelligence at De Montfort University, Leicester, UK. She graduated in Telecommunication Engineering in 2009 at she obtained her $\mathrm{PhD}$ in Artificial Intelligence in 2015 both from the University of Granada. In 2017, she was awarded by the European Commission with the Prestigious Marie Curie Individual Fellowship.

Her research interests focus on applying computational intelligence techniques for solving complex real-world network based applications under uncertainty in social networks and e-health scenarios.

Dr. Ureña has published more than 45 indexed contributions in both highly regarded international journals and international conferences in the area of Artificial Intelligence and Computer Science. Furthermore she contributes as associate editor to the Journal of Intelligent Fuzzy Systems (JCR 1.426) and Journal of Artificial Intelligence and systems.

She has international experience in both academia and top technological companies such as. For more information please have a look to her website www.raquelurena.net.

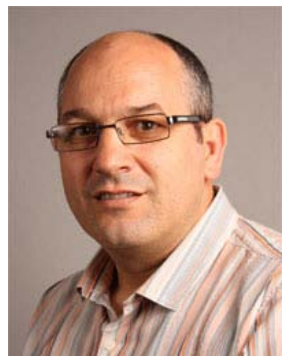

Francisco Chiclana received the B.Sc. and Ph.D. degrees in mathematics from the University of Granada, Granada, Spain, in 1989 and 2000, respectively.

He is currently a Professor of computational intelligence and decision making with the School of Computer Science and Informatics, Faculty of Technology, De Montfort University, Leicester, U.K., and an Honorary Professor with the Department of Mathematics, University of Leicester, Leicester, U.K., for a period from July 1, 2015 to June 30, 2018. He has published extensively in leading international journals with more than 70 journal articles indexed in Web of Science. He has also been consistently classed as one of the Most Cited Scientist in its field by ESI. His current research interests include decision making, computational intelligence, information fusion, consensus, rule induction, and sentiment analysis.

Dr. Chiclana is an Associate Editor of the IEEE Transactions on Systems, Man, and Cybernetics: Systems, Information Sciences, Soft Computing, the International Journal of Fuzzy Systems, and the Journal of Intelligent and Fuzzy Systems. He also serves as a member of the Editorial Board of Applied Soft Computing, Knowledge-Based Systems, and Multiple-Valued Logic and Soft Computing.

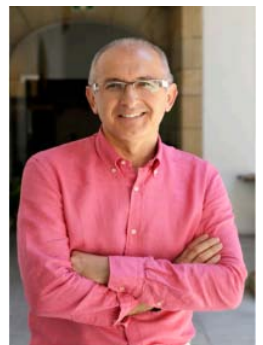

Enrique Herrera-Viedma received the M.Sc. and Ph.D. degrees in computer science from the University of Granada, Granada, Spain, in 1993 and 1996, respectively.

He is a Professor of computer science and the Vice-President for Research and Knowledge Transfer with University of Granada, Granada, Spain. Hish-index is 62 with more than 13000 citations received in Web of Science and 75 in Google Scholar with more than 24000 cites received. He has been identified as one of the world's most influential researchers by the Shanghai Center and Thomson Reuters/Clarivate Analytics in both computer science and engineering in the years 2014, 2015, 2016, and2017. His current research interests include group decision making, consensus models, linguistic modeling, aggregation of information, information retrieval, bibliometric, digital libraries, web quality evaluation, recommender systems, and social media.

Dr. Herrera-Viedma is a member of Government Board in SMC Society and an Associate Editor in several journals such as IEEE Transactions on Fuzzy Systems, IEEE Transactions on Systems, Man, and Cybernetics: Systems, IEEE Transactions on Intelligent Transport System, Information Sciences, Applied Soft Computing, Soft Computing, Fuzzy Optimization and Decision Making, and Knowledge-Based Systems. 